\title{
A Hybrid Algorithm for the J oint PDF Equation of Turbulent Reactive Flows
}

\author{
P. Jenny, S. B. Pope, M. Muradoglu, and D. A. Caughey \\ Sibley School of Mechanical and Aerospace Engineering, Cornell University, Ithaca, New York 14853 \\ E-mail: pjnn@chevron.com
}

Received July 19, 1999; revised June 13, 2000

In this paper a new particle-finite-volume hybrid algorithm for the joint velocityfrequency-composition PDF method for turbulent reactive flows is presented. This method is a combination of a finite-volume scheme and a particle method. The finitevolume scheme is used to solve the Reynolds averaged Navier-Stokes equations and the particle method to solve the joint PDF transport equation. The motivation is to reduce the bias and the statistical error and to have an algorithm which is more efficient than stand-alone particle-mesh methods. Therefore, in the particle method we use the smoother mean density $\langle\rho\rangle$ and Favre averaged velocity $\tilde{\mathbf{U}}$ fields computed by the finite-volume scheme: This scheme is an Euler solver for compressible flow with the turbulent fluxes and the reaction term, which are computed by the particle algorithm, as source terms. Since some of the quantities are computed twice (i.e., the mean density $\langle\rho\rangle$ and the Favre averaged sensible internal energy $\tilde{e}_{s}$ ), by the finite-volume scheme and by the particle method, the hybrid algorithm is redundant. Although the model differential equations are consistent, it was difficult to satisfy consistency numerically, and an accurate particle tracking algorithm is crucial. Therefore a new scheme to interpolate the Favre averaged velocity has been developed which is second-order accurate and quasi conservative; i.e., it is based on the fluxes at the volume interfaces. Another important issue is the coupling between the finite-volume scheme and the particle method. A new time-averaging technique adds stability to the hybrid algorithm, and it also reduces the bias and the statistical error enormously. The properties of the new algorithm are demonstrated by results for a nonpremixed piloted-jet flame test case. First it is shown that the solution becomes statistically stationary and that it is internally consistent. Studies of the asymptotic behavior show that, for a given error tolerance, the new hybrid algorithm requires much less computer time than the stand-alone particle-mesh method (for this pilotedjet flame test case a factor of 20 times less). Finally, grid convergence studies verify that the scheme is second-order accurate in space. (c) 2001 Academic Press

Key Words: PDF methods; particle methods; Monte Carlo; finite volume; turbulence modeling; nonpremixed combustion; piloted jet flames. 


\section{INTRODUCTION}

The calculation of complex turbulent reactive flows is of great importance for many engineering applications. The accuracy of such calculations depends mainly on turbulence models, combustion models, and the numerical algorithm. The task of turbulence modeling is to make the flow computations feasible without a great loss of accuracy. Reduced reaction mechanisms do the same for the chemistry. To solve the resulting set of equations accurate numerical algorithms are required. In the context of probability density function (PDF) methods, less attention has been paid to the last point, which is the motivation for this paper.

Traditional turbulence models, including two-equation models [20, 48] and secondmoment closures [19], are based on Reynolds averaging techniques and yield modeled equations for statistical moments. In comparison to these models, PDF methods achieve closure through a modeled transport equation for the one-point, one-time PDF of certain fluid properties in a turbulent flow $[8,30,31]$. The advantage of PDF methods is that both convection and reaction are represented exactly without modeling assumptions. The tremendous amount of statistical information contained in the PDFs obviously provides a fuller description of turbulent flows than two-equation models or second-moment closures. An overview of turbulence theory and modeling approaches is given in [38]. During the past decade, progress in PDF methods has been made from several aspects: adopting a more advanced joint velocity-frequency-composition PDF method which provides a model for the turbulent time scale $[39,45]$; incorporating modeling techniques developed for secondmoment closures $[10,32,33,46]$; and developing a computationally efficient scheme to treat detailed reaction chemistry [36]. These models have been successfully applied to modeling several inert flows [1, 6, 25], reactive flows, and turbulent flames [24, 27, 41].

Different numerical solution algorithms are required for turbulence models of different levels. Moment closures result in a set of partial differential equations. These equations are usually solved numerically by finite-difference or finite-volume methods [17]. In contrast to moment-closure model equations, the modeled PDF transport equation has a completely different structure. It is a high-dimensional scalar transport equation, and it is infeasible to solve it with a finite-volume or a finite-difference method. From early times in the development of PDF methods, Monte Carlo techniques in which the PDF is represented by an ensemble of particles have been employed [29]. Stochastic differential equations (SDEs) are constructed to model the particle properties, e.g., velocity, composition, and frequency, such that the particles exhibit the same PDF as in turbulent flows.

Monte Carlo methods are widely used in computational physics [18] to solve highdimensional problems since the computational costs increase only linearly with the number of dimensions. Their application in PDF methods has progressed through different stages. In the first method, the particles are located at grid nodes in physical space [29]. Pope [30] then suggested that it is preferable to use a method in which the particles are continuously distributed. Later a hybrid method was implemented in the code PDF $2 D S$ in which composition PDFs are calculated by Monte Carlo methods while a finite-volume method is applied to solve for the mean velocity, dissipation, and mean pressure fields [2, 5, 27]. More recently, a stand-alone particle-mesh algorithm has been developed for the joint velocity-frequency-composition PDF model [35]. This method is implemented in the code $P D F 2 D V$ [34]. This is a code to calculate statistically stationary two-dimensional (plane or axi-symmetric) turbulent reactive flows using the joint velocity-frequency-composition PDF method. It has been applied in several published calculations [1, 7, 9, 41, 47]. 
The motivation for the current work was to develop an algorithm for the joint velocityfrequency-composition PDF model which has less statistical and bias error than the previously developed stand-alone particle-mesh algorithms and is, therefore, more efficient. In the new method a finite-volume scheme is applied to solve the Reynolds averaged NavierStokes equations. The way the thermodynamic coupling is done is crucial, and the present approach was inspired by that of Colucci et al. [4]. The quantities extracted from the particle field and fed into the finite-volume scheme are the turbulent fluxes, the Favre averaged energy source term, and the ratio of the Favre averaged sensible enthalpy to the Favre averaged sensible internal energy. The particles in the particle method evolve according to the joint $P D F$ (JPDF) transport equation.

This work is contemporaneous with the parallel study of Muradoglu et al. [26]. A distinction between these works is the coupling method. Muradoglu et al. use a loose coupling approach in which each outer iteration consists of many iterations of the finite-volume solver followed by many time steps of the particle method. Here, in contrast, we use a tight coupling approach in which each outer iteration consists of a single finite-volume iteration and a single step of the particle method. This tightly coupled algorithm has been implemented in the code $P D F-2 D-F V$. Opposed to the studies in [26], this paper deals with an axi-symmetric test case of a nonpremixed piloted-jet flame and major improvements of the algorithm were necessary:

- Since it is crucial to achieve consistency between the finite volume data and the particle data a novel interpolation scheme for the mean velocity field has been developed and is described in Section 5.7 and Appendix A.

- To ensure that the mean of the fluctuating components of the particle velocities is $\approx 0$ (and is numerically consistent with the model) it is necessary to correct the fluctuating components of the particle velocities. The correction algorithm is explained in Section 5.8.

- The bias error can be reduced to an acceptable level by applying very large numbers of particles. This is a feasible approach if there are not too many cells, as in most 1D applications. In multidimensions, however, the sensitivity of the bias error on the number of particles determines mainly the efficiency of the PDF algorithm for a given level of numerical accuracy. Therefore a novel time-averaging technique has been developed and has proved to reduce the bias error dramatically (not only the statistical error as most other time-averaging schemes do). The time-averaging scheme is presented in Section 5.9 and explained in detail in Appendix B.

- In a systematic study the hybrid algorithm is carefully compared with an established particle mesh method in terms of numerical accuracy and efficiency (Section 6).

Like $P D F 2 D V$, the hybrid algorithm presented here simulates statistically stationary twodimensional (plane or axi-symmetric) turbulent reactive flows. The computational domain is divided into $M_{x} \times M_{y}$ cells, and the total number of particles is $N_{p}$. The modeled SDEs for the particle properties are solved by a pseudo-time marching scheme with time-step $\Delta t$. The primary numerical parameters in $P D F-2 D-F V$ calculations are $M_{x} \times M_{y}, \Delta t$, and $N_{p}$.

Since the focus of this work was to demonstrate the numerical behavior of the hybrid algorithm, the accuracy of the turbulence model and that of the combustion model have not been studied here. A flamelet model is used for the chemistry, but more detailed chemical reaction mechanisms have been used for PDF modeling studies, e.g., in [50].

In the next section the thermo-chemistry is explained. Section 3 introduces the PDF transport equation and shows how it is related to the Navier-Stokes equations and the 
Reynolds averaged Navier-Stokes equations. Section 4 explains how the unclosed terms in the JPDF transport equation are modeled, and in Section 5 the new algorithm to solve the modeled PDF transport equation is described. Finally results of a nonpremixed piloted-jet flame test case (in Section 6) demonstrate the numerical properties of the algorithm. In the appendices some of the numerical issues are explained in detail; in particular, in Appendix A the velocity interpolation scheme and in Appendix B the time-averaging technique are described.

\section{THERMO-CHEMISTRY}

We consider reactive and nonreactive flow of ideal gas mixtures. In this section the thermo-chemistry of an ideal gas mixture is described. Some nonstandard quantities are introduced to re-express the equation of state in a form suitable for the hybrid approach.

The thermo-chemical state of the fluid is characterized by the pressure $p$, the temperature $T$, and the mass fractions $\mathbf{Y}=\left(Y_{1}, Y_{2}, \ldots, Y_{N_{s}}\right)^{T}$ of the $N_{s}$ species. The molecular weight of species $\alpha$ is $W_{\alpha}$ and its gas constant is

$$
R_{\alpha}=\frac{\mathcal{R}}{W_{\alpha}},
$$

where $\mathcal{R}$ is the universal gas constant.

The thermodynamic database thermdat of the CHEMKIN package is used giving for each species $\alpha$ the values of the specific enthalpy of formation $h_{\alpha}^{0}$ at the reference temperature $T_{0}=298.15 \mathrm{~K}$ and the constant-pressure specific heat $c_{p \alpha}(T)$ as polynomial function of $T$. The specific sensible enthalpy is given by

$$
h_{s \alpha}(T)=\int_{0}^{T} c_{p \alpha}\left(T^{\prime}\right) d T^{\prime}=\bar{c}_{p \alpha}(T) T,
$$

where the (nonstandard) mean specific heat $\bar{c}_{p \alpha}(T)$ is defined by Eq. (2) to be

$$
\bar{c}_{p \alpha}(T)=\frac{1}{T} \int_{0}^{T} c_{p \alpha}\left(T^{\prime}\right) d T^{\prime} .
$$

The specific sensible internal energy is given by

$$
e_{s \alpha}(T)=\left(\bar{c}_{p \alpha}(T)-R_{\alpha}\right) T=\bar{c}_{v \alpha}(T) T,
$$

where $\bar{c}_{v \alpha}$ is defined similarly to $\bar{c}_{p \alpha}$. For the mixture, the specific sensible enthalpy is

$$
h_{s}(\mathbf{Y}, T)=\sum_{\alpha=1}^{N_{s}} Y_{\alpha} h_{s \alpha}(T)
$$

and similar equations define $e_{s}(\mathbf{Y}, T), \bar{c}_{p}(\mathbf{Y}, T), \bar{c}_{v}(\mathbf{Y}, T)$, and $R(\mathbf{Y})$. Furthermore, with $\mathbf{U}$ being the fluid velocity, the total sensible enthalpy is

$$
H_{s}(\mathbf{Y}, T)=h_{s}(\mathbf{Y}, T)+\frac{1}{2} U_{i} U_{i}
$$


and the total sensible energy is

$$
E_{s}(\mathbf{Y}, T)=e_{s}(\mathbf{Y}, T)+\frac{1}{2} U_{i} U_{i}
$$

The ideal gas law

$$
p=\rho R T
$$

where $\rho$ is the density, is applied. The above definition of $\bar{c}_{p}$ and $\bar{c}_{v}$ allows the equation of state to be written in terms of $e_{s}$ rather than $T$ as

$$
p=\rho R \frac{e_{s}}{\bar{c}_{v}}=\frac{\bar{c}_{p}-\bar{c}_{v}}{\bar{c}_{v}} \rho e_{s}=\left(\gamma^{\prime}-1\right) \rho e_{s},
$$

where $\gamma^{\prime}$ is defined by

$$
\gamma^{\prime}(\mathbf{Y}, T)=\frac{\bar{c}_{p}}{\bar{c}_{v}}=\frac{\bar{c}_{p}}{\bar{c}_{p}-R}
$$

Note that if $\bar{c}_{p}$ is independent of $T$, then $\gamma^{\prime}=\gamma=c_{p} / c_{v}$.

The net chemical reaction rate for species $\alpha$ is $S_{\alpha}$, defined so that, for a homogeneous mixture, the mass fractions evolve by

$$
\frac{d Y_{\alpha}}{d t}=S_{\alpha}(\mathbf{Y}, p, T) .
$$

Finally the reaction energy source term $\dot{Q}$ is

$$
\dot{Q}=-\sum_{\alpha=1}^{N_{s}} S_{\alpha}(\mathbf{Y}, p, T) h_{\alpha}^{0}
$$

For the present calculations we assume that $S_{\alpha}$ does not depend on the pressure.

\section{JOINT PDF FORMULATION}

In this section the JPDF transport equation and its relation to the Navier-Stokes equations and to the Reynolds averaged Navier-Stokes equations are explained. The basic idea of PDF methods is to describe the state of the flow at the location $\mathbf{x}=\left(x_{1}, x_{2}, x_{3}\right)$ at the time $t$ in terms of a one-point one-time Eulerian mass-weighted probability density function $\tilde{f}^{\prime}(\mathbf{V}, \Psi ; \mathbf{x}, t)$ of the velocity $\mathbf{U}=\left(U_{1}, U_{2}, U_{3}\right)^{T}$ and the composition variables $\boldsymbol{\Phi}=\left(\phi_{1}, \phi_{2}, \ldots, \phi_{N_{s}+1}\right)^{T}$ (species mass fractions and sensible enthalpy). The sample space variable corresponding to $\mathbf{U}$ is $\mathbf{V}=\left(V_{1}, V_{2}, V_{3}\right)^{T}$ and the sample space variable corresponding to $\boldsymbol{\Phi}$ is $\boldsymbol{\Psi}=\left(\psi_{1}, \psi_{2}, \ldots, \psi_{N_{s}+1}\right)^{T}$. The transport equation for $\tilde{f}^{\prime}(\mathbf{V}, \boldsymbol{\Psi} ; \mathbf{x}, t)$

$$
\begin{aligned}
& \langle\rho\rangle \frac{\partial \tilde{f}^{\prime}}{\partial t}+\langle\rho\rangle V_{j} \frac{\partial \tilde{f}^{\prime}}{\partial x_{j}}-\frac{\partial\langle p\rangle}{\partial x_{j}} \frac{\partial \tilde{f}^{\prime}}{\partial V_{j}}+\frac{\partial}{\partial \psi_{\alpha}}\left(\langle\rho\rangle S_{\alpha} \tilde{f}^{\prime}\right) \\
& =\frac{\partial}{\partial V_{j}}\left(\left\langle-\frac{\partial \tau_{i j}}{\partial x_{i}}+\frac{\partial p^{\prime}}{\partial x_{j}} \mid \mathbf{V}, \boldsymbol{\Psi}\right\rangle \tilde{f}^{\prime}\right)+\frac{\partial}{\partial \psi_{\alpha}}\left(\left\langle\frac{\partial J_{i}^{\alpha}}{\partial x_{i}} \mid \mathbf{V}, \Psi\right\rangle \tilde{f}^{\prime}\right)
\end{aligned}
$$


has been derived from the Navier-Stokes equations [30] and is exact (. denotes mass (Favre) averaged quantities and $\langle\cdot\rangle$ denotes volume (Reynolds) averaged quantities). Equation (13) is a scalar transport equation and must be solved in $N_{s}+8$ dimensions (velocity-compositionphysical space plus time). The first term is the time derivative of $\tilde{f}^{\prime}$, the second term is for the evolution of $\tilde{f}^{\prime}$ in the physical space, $\partial\langle p\rangle / \partial x_{j}$ is for the acceleration because of the mean pressure gradient, and the last term on the left-hand side evolves $\tilde{f}^{\prime}$ in the composition sample space due to chemical reactions. It is remarkable that all these terms are in closed form and do not have to be modeled. The terms which still have to be modeled in order to close Eq. (13) are those on the right-hand side, i.e., the effects of the viscous stress tensor $\tau_{i j}$ and of the fluctuating pressure gradients $\partial p^{\prime} / \partial x_{j}$ and the effect of the molecular diffusion fluxes $J_{i}^{\alpha}$ (of the scalar $\alpha$ in direction $x_{i}$ ). Notice that these are conditional probabilities.

For later explanations it is important to mention that the Reynolds averaged Navier-Stokes equations

$$
\begin{gathered}
\frac{\partial}{\partial t}\langle\rho\rangle+\frac{\partial}{\partial x_{i}}\left(\langle\rho\rangle \tilde{U}_{i}\right)=0 \\
\frac{\partial}{\partial t}\left(\langle\rho\rangle \tilde{U}_{i}\right)+\frac{\partial}{\partial x_{j}}\left(\langle\rho\rangle \tilde{U}_{i} \tilde{U}_{j}+\langle p\rangle \delta_{i j}\right)=-\frac{\partial}{\partial x_{j}}\left(\langle\rho\rangle \widetilde{u_{i} u_{j}}\right) \\
\frac{\partial}{\partial t}\left(\langle\rho\rangle \tilde{E}_{s}\right)+\frac{\partial}{\partial x_{i}}\left(\tilde{U}_{i}\left(\langle\rho\rangle \tilde{E}_{s}+\langle p\rangle\right)\right) \\
=\langle\rho \dot{Q}\rangle-\frac{\partial}{\partial x_{1}}\left(\langle\rho\rangle \widetilde{u_{i} h_{s}^{\prime \prime}}\right)-\frac{\partial}{\partial x_{i}}\left(\frac{\langle\rho\rangle}{2} \widetilde{u_{i}} \widetilde{u_{j} u_{j}}\right)-\frac{\partial}{\partial x_{i}}\left(\tilde{U}_{j}\langle\rho\rangle \widetilde{u_{i} u_{j}}\right)
\end{gathered}
$$

can be derived from (13) by integration. Because only high-Reynolds-number flow remote from walls is considered here, the molecular effects in the conservation equations are neglected. By definition $h_{s}^{\prime \prime}=h_{s}-\tilde{h}_{s}$ and $\mathbf{u}=\mathbf{U}-\tilde{\mathbf{U}}$. Finally the mean equation of state is given by

$$
\left\langle p^{\prime}\right\rangle=\left(\hat{\gamma}^{\prime}-1\right)\left(\langle\rho\rangle \tilde{E}_{s}-\frac{\langle\rho\rangle}{2}\left(\tilde{U}_{i} \tilde{U}_{i}+\widetilde{u_{i} u_{i}}\right)\right),
$$

where

$$
\hat{\gamma}^{\prime}=\frac{\tilde{h}_{s}}{\tilde{e}_{s}}
$$

\section{MODELED JPDF EQUATION}

To model also the turbulence frequency $\omega(\mathbf{x}, \mathrm{t})$ a modeled transport equation of the mass weighted joint velocity-frequency-composition PDF $\tilde{f}$ is solved [12, 45].

We define the mass density function (mdf) $\mathcal{F}$ and the one-point one-time Eulerian massweighted joint velocity-frequency-composition $\operatorname{PDF} \tilde{f}$ of $\mathbf{U}(\mathbf{x}, t), \mathbf{\Phi}(\mathbf{x}, t)$ and $\omega(\mathbf{x}, t)$ by

$$
\langle\rho\rangle \tilde{f}(\mathbf{V}, \Psi, \theta ; \mathbf{x}, t)=\mathcal{F}(\mathbf{V}, \Psi, \theta ; \mathbf{x}, t) \equiv \rho(\boldsymbol{\Psi})\langle\delta(\mathbf{U}-\mathbf{V}) \delta(\mathbf{\Phi}-\mathbf{\Psi}) \delta(\omega-\theta)\rangle,
$$

where $\theta$ is the sample space variable of $\omega$.

Because of the high-dimensional space in which $\tilde{f}$, evolves (together with $\theta$ the number of dimensions is $N_{s}+8$; e.g., in 3D with 20 species the number of dimensions is 28 ) it is 
infeasible to solve the modeled transport equation for $\tilde{f}$ using a finite-difference or finitevolume scheme. Fortunately with the Monte Carlo approach (Lagrangian view; particle method) the computational costs increase only linearly with the number of sample space dimensions, and therefore PDF simulations are feasible.

From a Largrangian viewpoint, the flow is represented by a set of particles which evolve by stochastic differential equations. This is done so that the particles exhibit the same JPDF as the solution of the modeled JPDF transport equation. Each particle has a set of properties $\left\{m^{*}, \mathbf{X}^{*}, \mathbf{U}^{*}, \Phi^{*}, \omega^{*}\right\}$, where $m^{*}$ represents the mass of the particle, $\mathbf{X}^{*}$ its coordinate, $\mathbf{U}^{*}$ the velocity, $\boldsymbol{\Phi}^{*}$ the composition vector, and $\omega^{*}$ the particle's turbulence frequency (the superscript $*$ denotes that the quantity is a particle property). For the evolution of $\mathcal{F}$, model equations have been developed, using the modeling theories for turbulent reactive flows. Models are required only for the pressure-strian-rate correlation, mixing, and dissipation. Models for particle velocity, turbulence frequency, and molecular mixing are described in the following subsections. These are not the most sophisticated models, but serve to illustrate the coupling issues addressed here.

\subsection{Velocity Model}

In PDF methods, the fluid particle velocity $\mathbf{U}^{+}(t)$ is represented by the stochastic particle velocity $\mathbf{U}^{*}(t)$ and various Langevin models have been developed to model the evolution of the particles in the velocity-sample-space $[10,30,32,46]$. Here we use the simplest one; the simplified Langevin model (SLM),

$$
d U_{i}^{*}(t)=-\frac{1}{\langle\rho\rangle} \frac{\partial\langle p\rangle}{\partial x_{i}} d t-\left(\frac{1}{2}+\frac{3}{4} C_{0}\right) \Omega\left(U_{i}^{*}(t)-\tilde{U}_{i}\right) d t+\left(C_{0} k \Omega\right)^{1 / 2} d W_{i},
$$

where

$$
\Omega \equiv C_{\Omega} \frac{\left\langle\rho^{*} \omega^{*} \mid \omega^{*} \geq \tilde{\omega}\right\rangle}{\langle\rho\rangle}
$$

is the conditional Favre averaged turbulence frequency;

$$
k=\frac{\widetilde{u_{i} u_{i}}}{2}
$$

is the turbulence kinetic energy; and $C_{0}$ and $C_{\Omega}$ are model constants (Table I). Diffusion process is represented by a Wiener process $\mathbf{W}(t)$, where $d W_{i}(t)=W_{i}(t+d t)-W_{i}(t)$ is

\section{TABLE I}

\section{Model Constants}

\begin{tabular}{lll}
\hline Constant & Value & \multicolumn{1}{c}{ Used in } \\
\hline$C_{0}$ & 2.1 & SLM \\
$C_{\Omega}$ & 0.6893 & Definition of $\Omega$ \\
$C_{\omega 1}$ & 0.56 & Turbulence frequency model \\
$C_{\omega 2}$ & 0.9 & Turbulence frequency model \\
$C_{3}$ & 1.0 & Turbulence frequency model \\
$C_{4}$ & 0.25 & Turbulence frequency model \\
$C_{\phi}$ & 2.0 & IEM mixing model \\
\hline
\end{tabular}


normally distributed with $\left\langle d W_{i}(t)\right\rangle=0$ and $\left\langle d W_{i}(t) d W_{j}(t)\right\rangle=d t \delta_{i j}$. The SLM is equivalent to Rotta's model at the second-moment-closure level.

In the numerical algorithm (presented in Section 5) a modeled transport equation for $\tilde{g}(\mathbf{v}, \Psi, \theta ; \mathbf{x}, t)$ is solved. The function $\tilde{g}$ is the JPDF in the fluctuating velocity-frequencycomposition space, and $\mathbf{v}$ is the sample space variable of the fluctuating (Favre) velocity u. In place of $\mathbf{U}^{*}(t)$ the fluctuating part $\mathbf{u}^{*}(t)$ of the particle velocity becomes a particle property. The following modified Langevin equation,

$$
d u_{i}^{*}(t)=\frac{1}{\langle\rho\rangle} \frac{\partial\left(\langle\rho\rangle \widetilde{u_{i} u_{j}}\right)}{\partial x_{j}} d t-u_{j}^{*} \frac{\partial \tilde{U}_{i}}{\partial x_{j}} d t-\left(\frac{1}{2}+\frac{3}{4} C_{0}\right) \Omega u_{i}^{*}(t) d t+\left(C_{0} k \Omega\right)^{1 / 2} d W_{i},
$$

has been derived from (18). In contrast to $\tilde{f}$, the modified JPDF $\tilde{g}$ contains no information about the mean velocity $\tilde{\mathbf{U}}$, but $\tilde{f}$ and $\tilde{g}$ are otherwise equivalent.

\subsection{Model for the Turbulence Frequency}

To close Eq. (21) or (18) a model for the conditional turbulence frequency is needed. In many PDF codes a model for the mean dissipation (e.g., the $k-\varepsilon$ model) is used to estimate $\Omega$. In the joint velocity-frequency-composition model the turbulence frequency $\omega^{*}$ is a particle property, and to account for external intermittency effects, Eq. (19) is used to estimate $\Omega$. The model constant $C_{\Omega}$ (Table I) is chosen such that $\Omega$ equals $\tilde{\omega}$ for fully devolped homogeneous turbulence. Using this approach the Favre averaged turbulence dissipation can be defined as

$$
\tilde{\varepsilon}=k \Omega \text {. }
$$

The stochastic model for $\omega^{*}(t)$ is

$$
d \omega^{*}(t)=-C_{3}\left(\omega^{*}-\tilde{\omega}\right) \Omega d t-S_{\omega} \Omega \omega^{*}(t) d t+\left(2 C_{3} C_{4} \tilde{\omega} \Omega \omega^{*}(t)\right)^{1 / 2} d W,
$$

where $C_{3}$ and $C_{4}$ are model constants (Table 1) [12,45], and $W(t)$ is a Wiener process, independent of that in the velocity model. In Eq. (23), $S_{\omega}$ is the source of turbulence frequency. Here it is modeled as

$$
S_{\omega}=C_{\omega 2}-C_{\omega 1} \frac{P}{k \Omega},
$$

where $P$ is the turbulence production

$$
P=-\widetilde{u_{i} u_{j}} \frac{\partial \tilde{U}_{i}}{\partial x_{j}}
$$

and $C_{\omega 1}$ and $C_{\omega 2}$ are further model constants (Table I).

\subsection{Mixing Model}

The Lagrangian approach is also used to model the scalar properties $\boldsymbol{\Phi}^{+}(t)$, i.e., $N_{s}$ mass fractions $Y_{\alpha}$ and the sensible enthalpy $h_{s}$, following a particle. That is, $\boldsymbol{\Phi}^{+}(t)$ is modeled by a stochastic process $\boldsymbol{\Phi}^{*}(t)$. The effects of molecular diffusion are accounted for by a mixing model. Here the simplest model, the IEM or LMSE model [8], is applied, so that the particle composition evolves by

$$
\frac{d \boldsymbol{\Phi}^{*}(t)}{d t}=-\frac{1}{2} C_{\phi} \Omega\left(\boldsymbol{\Phi}^{*}(t)-\tilde{\boldsymbol{\Phi}}\right)+\mathbf{S}\left(\boldsymbol{\Phi}^{*}(t)\right)
$$


where the standard model constant $C_{\phi}$ is used (Table I), and $\mathbf{S}$ is the source term due to the chemical reactions. Mixing models ae crucial in PDF calculations of turbulent nonpremixed flames with finite-rate kinetics. The IEM model is problematic in this respect $[37,43]$; however, good results are obtained when it is used in conjuction with equilibrium or flamelet models for near equilibrium nonpremixed flames [49], as is the case here.

For the species $\left(\alpha=1,2, \ldots, N_{s}\right)$ the source $S_{\alpha}$ in Eq. (26) is the reaction rate; for enthalpy $\left(\alpha=N_{s}+1\right)$ the source is

$$
S_{N_{s}+1}=-\sum_{\alpha=1}^{N_{s}} S_{\alpha} h_{\alpha}^{0} .
$$

\section{NUMERICAL SCHEME}

Although the Monte Carlo technique has proven to be an effective tool for solving the modeled JPDF evolution equation (Section 4), and JPDF methods are successfully applied to many flow problems of practical relevance, they are relatively expensive because many particles are required to keep the bias and the statistical error small. In the past, various strategies have been applied to solve the modeled JPDF transport equation (see Fig. 1). The idea of using a hybrid algorithm to reduce the statistical and the bias error is not new. Previous schemes are either only composition PDF methods or the modeled equations are inconsistent (e.g., in PDF2DS there are two values of the turbulent kinetic energy $k$; one computed by the $k-\varepsilon$ model and one computed by the PDF model). In PDF2DV, which is the implementation of a stand-alone particle-mesh method, the joint velocity-frequencycompositon PDF transport equation is solved, and there are no internal inconsistencies. Numerical issues of this stand-alone particle-mesh method are the following.

- Many particles per cell are required to keep the bias and statistical error small. This makes simulations expensive.

- A Poisson equation has to be solved for the mean pressure and contains damping and smoothing terms that make it quite complicated.

The goal of the present work was to devlop a hybrid scheme that solves for the joint velocityfrequency-composition PDF, is internally consistent, and is more efficient than stand-alone

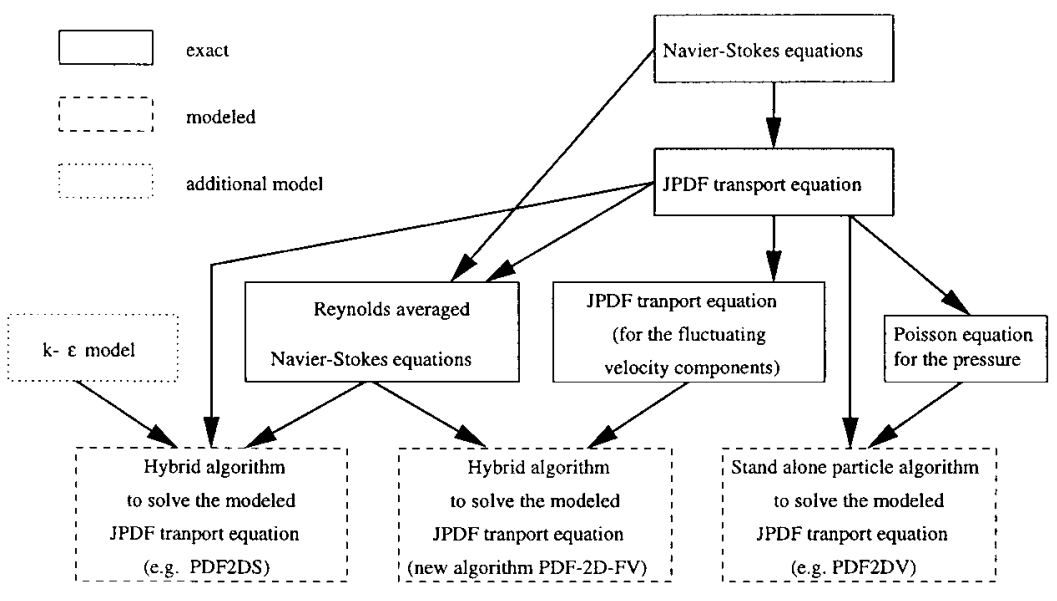

FIG. 1. Different PDF algorithms. 
particle-mesh methods. The new algorithm is implemented in the code $P D F-2 D-F V$ and has the following properties:

- The joint velocity-frequency-composition PDF evolution equation for turbulent reactive flow is solved.

- It is a combination of a finite-volume scheme and a particle method.

- The modeled equations solved by the two methods are consistent.

- Internal consistency is approximately satisfied on the numerical level.

- The bias and statistical errors are much smaller than those for stand-alone particle-mesh methods (for a given number of particles per cell).

- No Poisson equation for the mean pressure has to be solved.

- It is much more efficient than stand-alone particle-mesh methods.

In this section a general description of the $P D F-2 D-F V$ code is given. Like $P D F 2 D V$ the code is designed to model statistically stationary two-dimensional (plane or axisymmetric) turbulent reactive flows.

\subsection{Basic Idea}

On an empirical basis it has been found that in stand-alone particle-mesh methods the second term on the right-hand side of Eq. (18) is one of the main sources of the bias error. This term causes the particle velocity components $U_{i}^{*}$ to relax toward the mean $\tilde{U}_{i}$ which is extracted from the particle field itself. The present method does not have this problem since (21) is solved instead, where the fluctuating particle velocity components $u_{i}^{*}$ relax toward zero. In fact, we solve for the modeled JPDF $\tilde{g}(\mathbf{v}, \Psi, \theta ; \mathbf{x}, t)$, which is equivalent to $\tilde{f}(\mathbf{V}, \Psi, \theta ; \mathbf{x}, t)$, except that it contains no information about $\tilde{U}_{i}$. The mean velocity field is computed separately by a finite-volume scheme which solves the Reynolds averaged Navier-Stokes equations (14) (the right-hand side is computed by the particle code). Notice that $\tilde{f}(\mathbf{V}, \Psi, \theta ; \mathbf{x}, t)=\tilde{g}(\mathbf{V}-\tilde{\mathbf{U}}, \boldsymbol{\Psi}, \theta ; \mathbf{x}, t)$ and that the hybrid scheme is consistent on the level of the modeled equations. To have a stable, accurate, and consistent scheme it is crucial to satisfy this consistency numerically (notice that the coupled scheme is redundant; i.e., the mean density and the Favre averaged sensible internal energy are computed twice). Next a sketch of the hybrid algorithm is given, the properties of our finite-volume scheme are outlined, the particle method is explained, and some important coupling issues are discussed.

\subsection{Hybrid Algorithm}

Here a short outline of the new hybrid algorithm is given (Fig. 2). At the beginning the finite-volume and the particle data are initialized. Then the finite-volume scheme is advanced one pseudo time step (Section 5.3), and the Favre averaged velocity field and the mean density field are fed into the particle method. These mean fields are estimated at each particle position using an interpolation scheme (Section 5.7). In the particle code (Section 5.4), the particles evolve in the physical space, in the velocity sample space, in the frequency sample space, and in the composition sample space. To evolve the particles in the composition sample space as a result of chemical reactions, a chemical interface is called for each particle. From the new particle field the turbulent fluxes, the reaction energy source term, and $\hat{\gamma}^{\prime}$ are extracted (Section 5.4.1), and a time-averaging technique is applied (Section 5.9) to reduce the statistical and the bias error. Then the new time-averaged values 


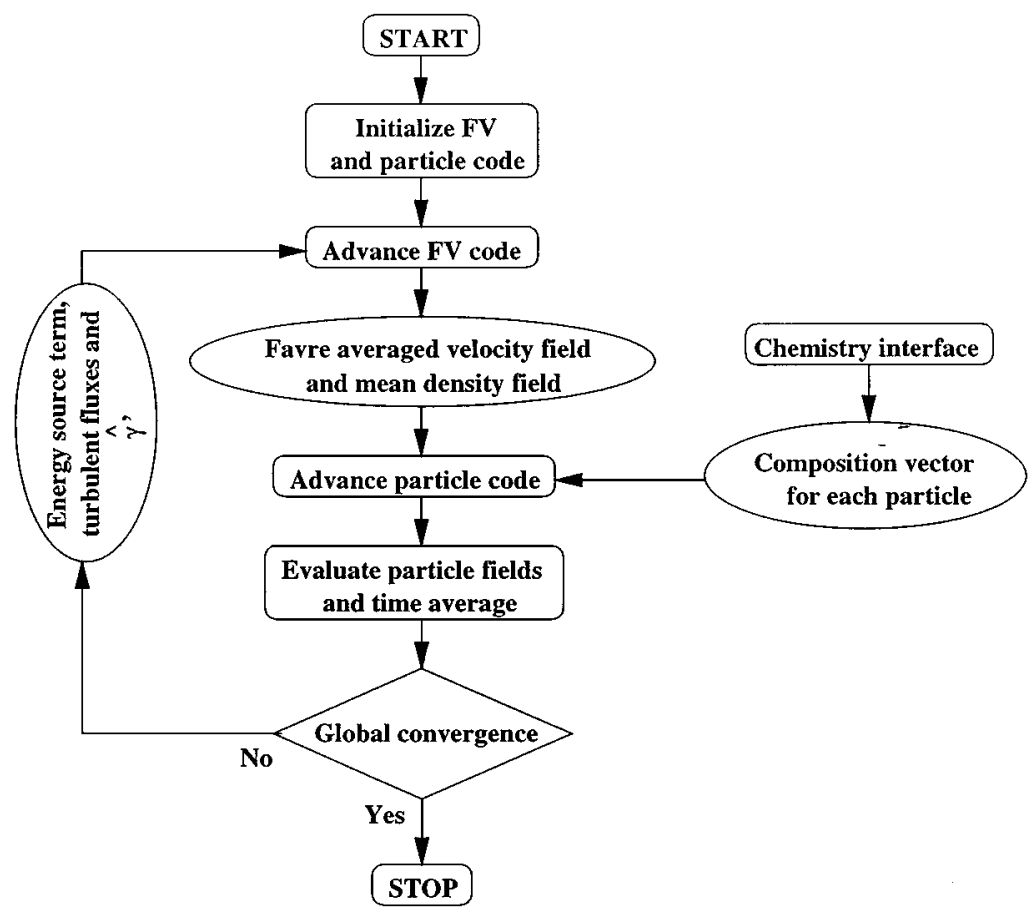

FIG. 2. Flow chart of the hybrid algorithm.

are fed into the finite-volume scheme (they are also used for the next particle method time step) and another time step starts. This loop is repeated until the solution has converged.

\subsection{Finite-Volume Scheme}

To solve the Reynolds averaged equations (14) a cell-centered finite-volume scheme for the inhomogeneous compressible Euler equations with source terms was implemented. It is a $2 \mathrm{D}$ (plane or axisymmetric) code which uses rectangular grids with the cells $(i, j)$ (Fig. 3). The cell centers are located at $\left(x_{1_{i}}, x_{2_{j}}\right)$, and the grid nodes are located at $\left(x_{1_{i+(1 / 2)}} x_{2_{j+(1 / 2)}}\right)$. The size of cell $(i, j)$ in the $x_{1}$ and $x_{2}$ directions is given by $\Delta x_{1_{i}}=$ $\left(x_{1_{i+(1 / 2)}}-x_{1_{i-(1 / 2)}}\right)$ and $\Delta x_{2_{j}}=x_{2_{j+(1 / 2)}}-x_{2_{j-(1 / 2)}}$, respectively. We use explicit local time stepping (the pseudo time step size $\Delta t$ is chosen according to the CFL stability criterion with a Courant number of 0.5) and a characteristic based Riemann solver is used for the computation of the fluxes at the volume interfaces [13, 42]. This solver is based on the idea of linearizing the Riemann invariants along the characteristics. For low-Mach-number flow, in spite of its simplicity, it is as accurate as more complicated and more expensive scheme such as Roe's approximate Riemann solver [40] (which does a much better job for high-Mach-number flows). Second-order spatial accuracy is obtained by applying the MUSCL scheme in combination with the minmod limiter [44]. All the other terms besides the inviscid fluxes are source terms extracted from the particle field. To avoid nonphysical oscillations in regions with nonconstant $\tilde{\gamma}^{\prime}(10)$ a correction scheme $[13,15,28]$ is applied. In order to overcome the stiffness problem at low Mach numbers a preconditioning technique, based on artificial reduction of the speed of sound, is used [13, 14]. 


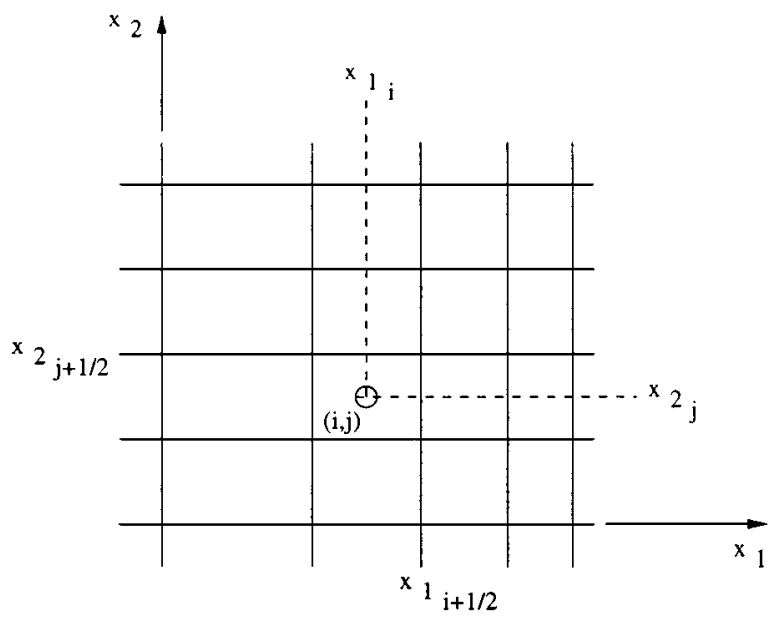

FIG. 3. Grid used in the finite-volume scheme and in the particle method.

\subsection{Particle Method}

In this section it is shown how mean fields are extracted from the particle data, how the particles evolve in the physical space, how the SDEs (21) and (23) are solved, and how the mixing model (26) is implemented. All this is done in the same way as in the code PDF2DV [34].

\subsubsection{Estimation of Means from the Particle Data}

Mean fields are used for three purposes:

- Some of the coefficients in the SDEs are mean fields.

- The source terms in the finite-volume scheme are cell averages of mean fields.

- Mean fields represent the results.

To represent mean fields we use the same rectangular grid with the cells $(i, j)$ as for the finite-volume method (Fig. 3). For the estimation and interpolation of mean fields we use different basis functions:

- To estimate mean particle properties at the grid node with the location $\left(x_{1_{i+(1 / 2)}}, x_{2_{j+(1 / 2)}}\right)$ the bilinear basis function $\hat{g}_{i+\frac{1}{2}, j+\frac{1}{2}}(\mathbf{x})$ is used (Fig. 4).

- The same bilinear basis function $\hat{g}_{i+\frac{1}{2}, j+\frac{1}{2}}(\mathbf{x})$ is used to interpolate properties from the grid nodes to the particle positions.

- To estimate mean particle properties in cell $(i, j)$ the top-hat function $\hat{\hat{g}}_{i, j}(\mathbf{x})$ is used, which is 1 for $\mathbf{x}$ in cell $(i, j)$ and 0 otherwise.

It is important to mention that at any location $\mathbf{x}$ within the computational domain the sum of all components of each basis function ( $\hat{g}$ and $\hat{\hat{g}}$, respectively) is one. Table II shows which means are extracted from the particle field. Favre averaged means at the grid nodes such as $\widetilde{u_{\ell} u_{k}}$ are estimated from the particle field as

$$
\left(\widetilde{u_{\ell} u_{k}}\right)_{i+\frac{1}{2}, j+\frac{1}{2}} \approx \frac{\sum_{n=1}^{N_{p}}\left(\hat{g}_{i+\frac{1}{2}, j+\frac{1}{2}}\left(\mathbf{X}^{*}\right) m^{*} u_{\ell}^{*} u_{k}^{*}\right)_{n}}{\sum_{n=1}^{N_{p}}\left(\hat{g}_{i+\frac{1}{2}, j+\frac{1}{2}}\left(\mathbf{X}^{*}\right) m^{*}\right)_{n}}
$$




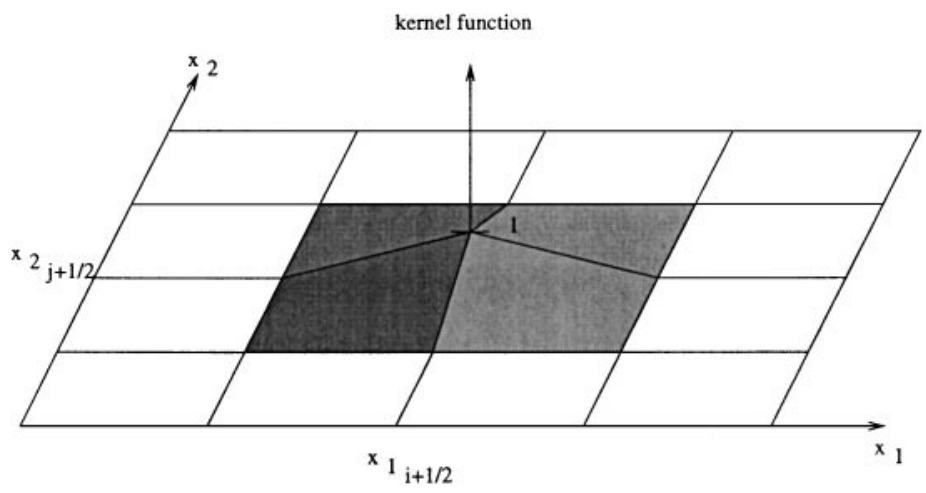

FIG. 4. Bilinear basis function $\hat{g}_{i+\frac{1}{2}, j+\frac{1}{2}}$ for kernel estimation and interpolation.

and for mean quantities at the cell centers such as $\langle\rho \dot{Q}\rangle$ the approximation

$$
\langle\rho \dot{Q}\rangle_{i, j} \approx-\frac{\sum_{n=1}^{N_{p}}\left(\hat{\hat{g}}_{i, j}\left(\mathbf{X}^{*}\right) m^{*} h_{\alpha}^{0} \frac{d}{d t} Y_{\alpha}^{*}\right)_{n}}{\sum_{n=1}^{N_{p}}\left(\hat{\hat{g}}_{i, j}\left(\mathbf{X}^{*}\right) m^{*}\right)_{n}}
$$

is used. Derivatives at the grid nodes such as $\partial\left(\langle\rho\rangle \widetilde{u_{\ell} u_{k}}\right) / \partial x_{1}$ are obtained by central differences as follows:

$$
\left(\frac{\partial\left(\langle\rho\rangle \widetilde{u_{\ell} u_{k}}\right)}{\partial x_{1}}\right)_{i+1 / 2, j+1 / 2} \approx \frac{\left(\langle\rho\rangle \widetilde{u_{\ell} u_{k}}\right)_{i+3 / 2, j+1 / 2}-\left(\langle\rho\rangle \widetilde{u_{\ell} u_{k}}\right)_{i-1 / 2, j+1 / 2}}{x_{1_{i+3 / 2, j+1 / 2}}-x_{1_{i-1 / 2, j+1 / 2}}} .
$$

The Reynolds stresses $\widetilde{u_{i} u_{j}}$, the scalar fluxes $\widetilde{u_{i} h_{s}^{\prime \prime}}, \tilde{\gamma}^{\prime}$, and the energy source term $\langle\rho \dot{Q}\rangle$ have to be estimated to close the system (14). All the other quantities in Table II, i.e.,

$$
\frac{1}{\langle\rho\rangle} \frac{\partial\left(\langle\rho\rangle \widetilde{u_{i} u_{j}}\right)}{\partial x_{j}}, \quad \Omega, \quad \tilde{\omega}, \quad k, \quad \text { and } \tilde{\boldsymbol{\Phi}}
$$

are used to close the particle evolution equations.

\section{TABLE II}

Means Extracted from the Particle Field

\begin{tabular}{clll}
\hline Quantity & Location & Equation & \multicolumn{1}{c}{ Purpose } \\
\hline$\widetilde{u_{i} u_{j}}$ & Grid node & $(27)$ & Finite-volume scheme, particle method \\
$\widetilde{u_{i} h_{s}^{\prime \prime}}$ & Grid node & $(27)$ & Finite-volume scheme \\
$\tilde{\omega}$ & Grid node & $(27)$ & Particle method \\
$\Omega$ & Grid node & $(27)$ & Particle method \\
$\frac{k}{\partial\langle\rho\rangle u_{i} u_{j}}$ & Grid node & $(27)$ & Particle method \\
$\left\langle\rho x_{k}\right.$ & Grid node & $(29)$ & Particle method \\
$\tilde{h_{s}}$ & Cell center & $(28)$ & Finite-volume scheme \\
$\tilde{e_{s}}$ & Cell center & $(28)$ & Finite-volume scheme \\
$\hat{\boldsymbol{\Phi}}$ & Cell center & $(28)$ & Finite-volume scheme \\
\hline
\end{tabular}




\subsubsection{Particle Motion}

A particle with the coordinate $\mathbf{X}^{*}$ evolves according to

$$
\frac{d \mathbf{X}^{*}(t)}{d t}=\tilde{\mathbf{U}}\left(\mathbf{X}^{*}(t), t\right)+\mathbf{u}^{*}(t)
$$

where $\tilde{\mathbf{U}}$ is computed by the finite-volume scheme at the volume interfaces and then interpolated to the particle position $\mathbf{X}^{*}$ (Section 5.7). To achieve second-order accuracy we apply the midpoint rule [34]. The first half step

$$
\mathbf{X}^{*^{n+\frac{1}{2}}}=\mathbf{X}^{*^{n}}+\frac{\Delta t}{2}\left(\tilde{\mathbf{U}}^{n}\left(\mathbf{X}^{*^{n}}\right)+\mathbf{u}^{*^{n}}\right)
$$

is performed to approximate the midpoint $\mathbf{X}^{*^{n+(1 / 2)}}$. The superscript $n$ denotes the old time level and $n+1$ the new time level. Then $\mathbf{u}^{*^{n+1}}$ is computed at the midpoint and used to perform the time step

$$
\mathbf{X}^{*^{n+1}}=\mathbf{X}^{*^{n}}+\Delta t\left(\tilde{\mathbf{U}}^{n}\left(\mathbf{X}^{*^{n+(1 / 2)}}\right)+\frac{1}{2}\left(\mathbf{u}^{*^{n}}+\mathbf{u}^{*^{n+1}}\right)\right)
$$

to get the new particle position $\mathbf{X}^{*^{n+1}}$.

\subsubsection{Simplified Langevin Model}

Defining

$$
\begin{aligned}
a_{i} & =\frac{1}{\langle\rho\rangle} \frac{\partial\left(\langle\rho\rangle \widetilde{u_{i} u_{j}}\right)}{\partial x_{j}} \\
b_{i j} & =-\frac{\partial \tilde{U}_{i}}{\partial x_{j}}-\left(\frac{1}{2}+\frac{3}{4} C_{0}\right) \Omega \delta_{i j} \quad \text { and } \\
c & =C_{0} \Omega k
\end{aligned}
$$

Eq. (21) becomes

$$
d u_{i}^{*}(t)=a_{i} d t+b_{i j} u_{j}^{*} d t+c^{1 / 2} d W_{i}
$$

To solve (34) numerically we apply the following second-order scheme [10, 34]

$$
\begin{aligned}
& \Delta u_{i}^{*}=\left(a_{i}+b_{i j} u_{j}^{*^{n}}\right) \Delta t+(c \Delta t)^{1 / 2} \xi_{i} \\
& u_{i}^{*^{n+1}}=u_{i}^{*^{n}}+\Delta u_{i}^{*}+\frac{1}{2} b_{i j} \Delta u_{j}^{*} \Delta t
\end{aligned}
$$

where $\xi_{i}$ is a random variable with standard normal distribution. The coefficients $a_{i}, b_{i j}$, and $c$ are evaluated at the midpoint. 


\subsubsection{Turbulence Frequency Model}

With

$$
\begin{aligned}
A & =C_{3} \tilde{\omega} \Omega \\
B & =\left(C_{3}+S_{\omega}\right) \Omega \\
C & =2 C_{3} C_{4} \tilde{\omega} \Omega,
\end{aligned}
$$

we write (23) as

$$
d \omega^{*}=A d t-B \omega^{*} d t+\left(C \omega^{*}\right)^{1 / 2} d W .
$$

For the numerical integration of Eq. (36) the coefficients $A, B$, and $C$ are considered to be frozen during one time step (evaluated at the midpoint) [34]. Then exact expressions for the mean and variance of $\omega^{*}(t+\Delta t)$ conditioned on $\omega^{*}(t)=\omega^{*^{n}}$ are

$$
X=\omega^{*^{n}} e^{-B \Delta t}+\frac{A}{B}\left(1-e^{-B \Delta t}\right)
$$

and

$$
\sigma^{2}=\frac{C \Delta t}{2(1+B \Delta t)}\left(X+\omega^{*^{n}}\right)
$$

Thus the new particle frequency is set to

$$
\omega^{*^{n+1}}=\max (0, X+\sigma \xi),
$$

where $\xi$ is a random variable with standard normal distribution, independent of that in the velocity model. The max-function in (39) is to guarantee realizability, and, as may be verified, it does not prevent the convergence of the method as $\Delta t$ tends to zero.

\subsubsection{IEM Mixing Model}

The ideal implementation of the IEM mixing model (26) has the following properties:

1. It guarantees realizability (boundedness).

2. It conserves the mean.

3. It is accurate in time.

4. It is spatially accurate.

The implementation described here (which follows [34]) is ideal with respect to 1, 2, and 3 , and it has the spatial accuracy of cloud-in-cell (CIC) [11]. For the $i$ th particle with composition vector $\boldsymbol{\Phi}_{i}^{*}(t)$, position $\mathbf{X}_{i}^{*}$ and mass $m_{i}^{*}$, the IEM model is

$$
d \boldsymbol{\Phi}_{i_{\text {mix }}}^{*}=-\frac{1}{2} C_{\phi} \Omega_{i}\left(\Phi_{i}^{*}-\tilde{\boldsymbol{\Phi}}_{i}\right) d t
$$

where $\Omega_{i}$ is the mean conditional frequency (24) at $\mathbf{X}_{i}^{*}$, and $\tilde{\boldsymbol{\Phi}}_{i}$ is the Favre averaged $\boldsymbol{\Phi}$ at $\mathbf{X}_{i}^{*}$. Additionally to (40) the particles evolve in the composition sample space because 
of chemical reactions. The essence of the implementation is to specify a valid numerical approximation $\hat{\boldsymbol{\Phi}}_{i}$ to $\tilde{\boldsymbol{\Phi}}_{i}$ such that the implementation of (40) has the properties listed above. With $\Omega_{i}$ and $\hat{\boldsymbol{\Phi}}_{i}$ frozen, the exact increment in $\boldsymbol{\Phi}_{i}^{*}$ over a time $\Delta t$ is

$$
\delta \boldsymbol{\Phi}_{i}^{*}=-d_{i}\left(\boldsymbol{\Phi}_{i}^{*}-\hat{\boldsymbol{\Phi}}_{i}\right)
$$

where

$$
d_{i}=1-e^{-\frac{1}{2} C_{\phi} \Omega_{i} \Delta t}
$$

Let $\mathbf{F}_{\alpha}$ be the value of $\hat{\boldsymbol{\Phi}}$ at the grid node $\alpha$ and let $\hat{g}_{\alpha}\left(\mathbf{X}_{i}^{*}\right)$ be the linear basis function coefficient, so that interpolation from the corners $\alpha$ to the particle position $\mathbf{X}_{i}^{*}$ yields

$$
\hat{\mathbf{\Phi}}_{i}=\sum_{\alpha} \hat{g}_{\alpha}\left(\mathbf{X}_{i}^{*}\right) \mathbf{F}_{\alpha}
$$

where

$$
1=\sum_{\alpha} \hat{g}_{\alpha}\left(\mathbf{X}_{i}^{*}\right)
$$

Thus

$$
\delta \boldsymbol{\Phi}_{i}^{*}=-d_{i}\left(\boldsymbol{\Phi}_{i}^{*}-\sum_{\alpha} \hat{g}_{\alpha}\left(\mathbf{X}_{i}^{*}\right) \mathbf{F}_{\alpha}\right)
$$

Now the global change of $\boldsymbol{\Phi}$ is

$$
\delta \mathbf{G} \equiv \sum_{i} m_{i}^{*} \delta \boldsymbol{\Phi}_{i}^{*}=-\sum_{i} m_{i}^{*} d_{i}\left(\boldsymbol{\Phi}_{i}^{*}-\sum_{\alpha} \hat{g}_{\alpha}\left(\mathbf{X}_{i}^{*}\right) \mathbf{F}_{\alpha}\right)
$$

Using (44), the right-hand side can be rewritten

$$
\delta \mathbf{G}=\sum_{\alpha}\left(-\left(\sum_{i} \hat{g}_{\alpha}\left(\mathbf{X}_{i}^{*}\right) m_{i}^{*} d_{i} \boldsymbol{\Phi}_{i}^{*}\right)+\mathbf{F}_{\alpha}\left(\sum_{i} \hat{g}_{\alpha}\left(\mathbf{X}_{i}^{*}\right) m_{i}^{*} d_{i}\right)\right) .
$$

Evidently a sufficient definition for $\mathbf{F}_{\alpha}$ to satisfy $\delta \mathbf{G}=0$, i.e., to have a conservative implementation, is given by

$$
\mathbf{F}_{\alpha}=\frac{\sum_{i} \hat{g}_{\alpha}\left(\mathbf{X}_{i}^{*}\right) m_{i}^{*} d_{i} \boldsymbol{\Phi}_{i}^{*}}{\sum_{i} \hat{g}_{\alpha}\left(\mathbf{X}_{i}^{*}\right) m_{i}^{*} d_{i}}
$$

This is the CIC mean with particle weighting $m_{i}^{*} d_{i}$ [34].

In summary, IEM is implemented via Eq. (41), with $d_{i}, \hat{\boldsymbol{\Phi}}_{i}$, and $\mathbf{F}_{\alpha}$ being defined by Eq. (42), (43), and (48). 


\subsection{Consistency Issues}

It has already been mentioned that the new algorithm is consistent at the level of the modeled equations. That is, if these equations were solved exactly (without numerical error), then the fields that are represented in both the finite-volume and particle methods would be identical. But it is difficult to satisfy consistency numerically. The mean density and the Favre averaged sensible internal energy fields are computed twice and therefore the algorithm is redundant (Section 5.1). First we define the mean particle mass density,

$$
q(\mathbf{x}, t)=\left\langle\sum_{i} m_{i}^{*} \delta\left(\mathbf{X}_{i}^{*}(t)-\mathbf{x}\right)\right\rangle .
$$

Since $q$ represents the mean fluid density $\langle\rho\rangle$ it is a consistency requirement that $q=\langle\rho\rangle$. In addition to an accurate interpolation (Section 5.7) of $\tilde{\mathbf{U}}$ from the finite-volume data to the particle positions, it is required that $\left\langle u_{i}^{*} \mid \mathbf{x}\right\rangle=0$ everywhere (Section 5.8). An additional consistency requirement is that the Favre averaged sensible internal energy $\tilde{e}_{s}$ computed by the finite-volume scheme corresponds well to the one computed by the particle method. Therefore, besides tracking the particles accurately, the scalar fluxes $\left\langle u_{i}^{*} h_{s}^{\prime \prime *} \mid \mathbf{x}\right\rangle$, which are fed into the finite-volume scheme, have to be accurate.

\subsection{Coupling}

In Section 5.2, a sketch of the new hybrid algorithm was shown; in Section 5.3, the properties of the finite-volume scheme were outlined; and in Section 5.4, it was described how mean quantities are extracted from the particle data and how the particle evolution equations are solved. Here we discuss the coupling of the two schemes, the finite-volume scheme to solve the Reynolds averaged Navier-Stokes equations and the particle method to model the evolution of $\tilde{g}$. Table III shows which information has to be exchanged between the two parts of the algorithm. Next it is described how the Favre averaged velocity $\tilde{\mathbf{U}}$ is interpolated from the finite-volume data to the particle positions and how it is achieved that $\left\langle\mathbf{u}^{*} \mid \mathbf{x}\right\rangle$ remains zero.

\subsection{Velocity Field Interpolation}

In Section 5.5 it was pointed out that an accurate scheme for the interpolation of $\tilde{\mathbf{U}}$ from the finite-volume data to the particle positions is required in order to have good agreement between the mean particle mass density $q$ and the mean fluid density $\langle\rho\rangle$ which is computed

TABLE III

Information Exchange Between the Particle System and the Finite-Volume Scheme

\begin{tabular}{lc}
\hline Information flow direction & Information \\
\hline $\begin{array}{l}\text { From the particle method } \\
\text { to the finite-volume scheme }\end{array}$ & $\widetilde{u_{i} u_{j}}, \widetilde{u_{i} h_{s}^{\prime \prime}},\langle\rho \dot{Q}\rangle$, \\
$\begin{array}{l}\text { From the finite-volume scheme } \\
\text { to the particle method }\end{array}$ & $\hat{\gamma}^{\prime}=\widetilde{h_{s}}$ \\
$\tilde{e}_{s}$ & $\tilde{\mathbf{U}},\langle\rho\rangle$ \\
\hline
\end{tabular}


by the finite-volume scheme. We have developed an interpolation scheme which is based on the fact that in a flow with a velocity field $\mathbf{U}(\mathbf{x}, t)$ the volume $d V(t)$ of an infinitesimal material volume evolves by

$$
\frac{d}{d t} \ln d V(t)=\nabla \cdot \mathbf{U}
$$

and the fluid density by

$$
\frac{D \ln \rho}{D t}=-\nabla \cdot \mathbf{U}
$$

Similarly, the expected mass density $q$ of particles evolves by

$$
\left(\frac{\partial}{\partial t}+\tilde{\mathbf{U}} \cdot \nabla\right) \ln q=-\nabla \cdot \tilde{\mathbf{U}} .
$$

Thus the dilatation field $\nabla \cdot \tilde{\mathbf{U}}$ experienced by the particles is of fundamental importance in particle methods. Our particles move with velocity $\tilde{\mathbf{U}}+\mathbf{u}^{*}$. For such particles it can be shown that (51) applies [30, 38].

For constant density flow $\nabla \cdot \tilde{\mathbf{U}}$ is zero, and in the steady-state for variable $\langle\rho\rangle$ we have $\nabla \cdot(\langle\rho\rangle \tilde{\mathbf{U}})=0$, which is satisfied in a weak form using cell centered finite-volume methods Therefore it makes most sense to use the mean velocities at the volume interfaces (computed by the flux solver) for the interpolation to the particle positions.

Our new interpolation scheme has the following properties:

- Within one cell the representation of $\tilde{U}_{1}$ is quadratic in $x_{1}$ and linear in $x_{2}$, and correspondingly the representation of $\tilde{U}_{2}$ is quadratic in $x_{2}$ and linear in $x_{1}$.

- The dilatation field $\nabla \cdot \tilde{\mathbf{U}}$ varies bilinearly with $x_{1}$ and $x_{2}$ within each cell.

- For constant density flow in the steady state the interpolated Favre averaged velocity field fulfills exactly $\nabla \cdot \tilde{\mathbf{U}}=0$ everywhere.

- The interpolated component $U_{i}$ is continuous in the $x_{i}$ direction, but in general not continuous in the $x_{j}$ direction, if $j \neq i$. This is a compromise necessary to avoid oscillations and to satisfy other requirements.

All these issues are discussed further in Appendix A, where the interpolation scheme is explained.

\subsection{Velocity Correction in the Nondeterministic Case}

With the velocity interpolation scheme presented in Appendix A we approximately achieve consistency between the mean particle mass density $q$ and the mean fluid density $\langle\rho\rangle$, if the flow is laminar, i.e., if the particle property $\mathbf{u}^{*}$ is zero for all particles. In the nondeterministic case, i.e., if $\mathbf{u}^{*}$ is not zero for all particles, an additional requirement besides an accurate interpolation of the Favre averaged velocity field is that the expectation of the fluctuating velocity components $\tilde{\mathbf{u}}$, i.e., $\left\langle\mathbf{u}^{*} \mid \mathbf{x}\right\rangle$, remains zero everywhere. To achieve this we correct $\mathbf{u}^{*}$ by subtracting the time average of the precorrected mean $\tilde{\mathbf{u}}\left(\mathbf{X}^{*}\right)$ (time averaging is explained in Section 5.9) after each time step.

\subsection{Time Averaging}

To achieve a stable scheme and to reduce the statistical and the bias error of a quantity $\tilde{\beta}$, which stands, for example, for $\widetilde{u_{i} u_{k}}, \widetilde{u_{i} h_{s}^{\prime \prime}},\langle\rho \dot{Q}\rangle$, or $\hat{\gamma}^{\prime}$, we use the following time-averaging 
technique,

$$
\tilde{\beta}^{n+1}=\mu \tilde{\beta}^{n}+(1-\mu) \tilde{\beta}_{i}^{n+1} \quad \text { with } \quad(0 \leq \mu<1),
$$

where

$$
\mu=\frac{K-1}{K} \quad \text { with the time-averaging factor } K \geq 1 .
$$

The significance of the time-averaging factor $K$ is that $K \Delta t$ is the characteristic timeaveraging time, and thus $K$ expresses this time as a number of steps. The subscript $i$ indicates the instantaneous value, and the superscripts $n$ and $n+1$ denote the old and new time levels, respectively. The new value $\tilde{\beta}^{n+1}$ is determined from the old value $\tilde{\beta}^{n}$ weighted with the factor $\mu$ and the instantaneous value $\tilde{\beta}_{i}^{n+1}$ weighted with $1-\mu$. In Appendix B a model system for studying the coupled system is analyzed. It is important to mention that the time-averaged quantities are also used in the SDEs.

\section{TEST CASE: NONPREMIXED PILOTED-JET FLAME}

This test case is an axisymmetric nonpremixed piloted-jet flame for which there exist experimental data [21, 22] and results from other PDF simulations [49]. The purpose is to compare the new hybrid scheme (implemented in the code $P D F-2 D-F V$ ) with the standalone particle-mesh method (implemented in the code PDF2DV [34]) in terms of accuracy and efficiency. We chose the same grids, the same initial and boundary conditions, the same turbulence models, and the same flamelet model used by $\mathrm{Xu}$ and Pope for their simulations [49]. It is important to mention here that the conclusions from the numerical studies are likely to be independent of the combustion model. An accurate description of the numerical test case is given in their paper.

A sketch of the burner used in the experiments on this flame is published in [22]. An axisymmetric jet of methane fuel with radius $R_{\text {jet }}=3.6 \mathrm{~mm}$ is centered in an annular pilot $\left(R_{\text {pilot }}=9 \mathrm{~mm}\right)$. The pilot burns a mixture of stoichiometric composition and provides a heat source to stabilize the main jet at the exit plane. The flame is accompanied by an unconfined coflow stream of air. The bulk velocity in the jet is specified to be $U_{\text {jet }}=41 \mathrm{~m} / \mathrm{s}$, the pilot has a velocity $U_{\text {pilot }}=24 \mathrm{~m} / \mathrm{s}$, and the coflow velocity is $U_{\text {coflow }}=15 \mathrm{~m} / \mathrm{s}$. These conditions correspond to the flame $L$ in Masri et al. [23]. Measurements have been performed for temperatures using thermocouples, velocity by LDA, and compositions by sample probes. Experimental data are published by Masri et al. [22] and are also available at the FTP site (Internet) of the University of Sydney [21].

Although the agreement between the experimental data and the computational results is not emphasized in this study, the Favre averaged velocity and mixture fraction profiles $40 R_{\text {jet }}$ downstream of the nozzle are shown in Fig. 5 and compared with experimental data by Masri et al. [22] and with results of a simulation with the code $P D F 2 D V[34,49]$. For both simulations the same $40 \times 40$ grid has been used, and in comparison to the previous computations $[3,24,41]$ these results are quite satisfactory considering the simple velocity model, mixing model, and thermo-chemistry used.

\subsection{Convergence Results}

Numerical experiments are conducted to isolate the statistical, the time stepping, the bias, and the discretization error. The statistical error in the results is reduced by time averaging. 

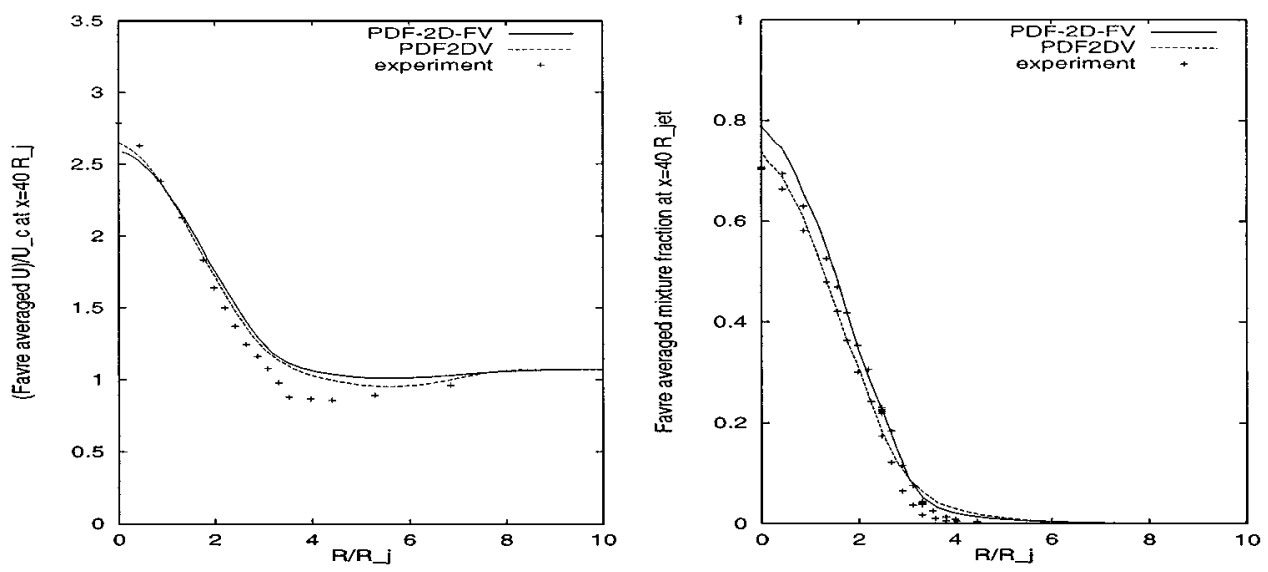

FIG. 5. Comparison of Favre averaged velocity and mixture fraction profiles at $x=40 R_{\text {jet }}$ (experimental data $[21,22]$, numerical results with $P D F 2 D V[34,49]$, and numerical results with $P D F-2 D-F V$; for the simulations the same $40 \times 40$ grid has been used).

The particle time steps $\Delta t$ fulfill the criterion $\Delta t \leq \min \left(\Delta x_{1} / U_{1}^{*}, \Delta x_{2} / U_{2}^{*}, 1 / \Omega\right) / 2$, and no differences in the results of simulations with smaller particle time steps could be observed. Also $\mathrm{Xu}$ and Pope [49] showed that the time-stepping error is negligible if the time step size satisfies the CFL condition and is smaller than the turbulence time scale. The numerical parameter for studying the bias error is the average number of particles per cell $N_{p c}$ in combination with the time-averaging factor $K$. For the spatial discretization error the relevant parameter is the number of cells $M^{2}$ : The grid spacings vary as $\Delta x \sim M^{-1}, \Delta y \sim M^{-1}$.

The stationarity of numerical solutions is first inspected. Next the bias error is investigated, and finally the spatial discretization error is analyzed.

\subsection{Stationary Solution}

Like $P D F 2 D V$, the new hybrid code $P D F-2 D-F V$ is designed to treat statistically stationary flows, such as the piloted-jet flame. In Fig. 6 the residual of the finite-volume part of the code is shown as a function of time steps for two different numbers of particles per cell $N_{p c}$ and for two different time-averaging factors $K$ (for the piloted-jet flame test case with a $40 \times 40$ grid). The two lowest curves represent the convergence histories of two simulations with the same $C_{b i a s}=N_{p c} K=4000$ (the bias error is the same for the same $C_{\text {bias }}$; Section 6.4), but with $N_{p c}=10$ and $N_{p c}=40$, respectively. It may be seen that in each case the residual generally decreases over the first 3000-6000 time steps, and then it achieves statistical stationarity. In the statistically stationary state, the levels of the residuals are determined by the statistical fluctuations arising from the particle code. The simulation with $N_{p c}=10$ reaches the statistically stationary state in 6000 time steps (about 1 hour on a $400-\mathrm{MHz}$ Pentium machine), and the simulation with $N_{p c}=40$ requires 4000 time steps (almost 3 hours on a 400-MHz Pentium machine).

\subsection{Internal Consistency}

Here it is shown that the mean density $\langle\rho\rangle$ field from the finite-volume data and the mean particle mass density $q$ field are approximately consistent. In Fig. 7 contour lines of these two fields are shown in the same plot, and a very good agreement can be observed. 


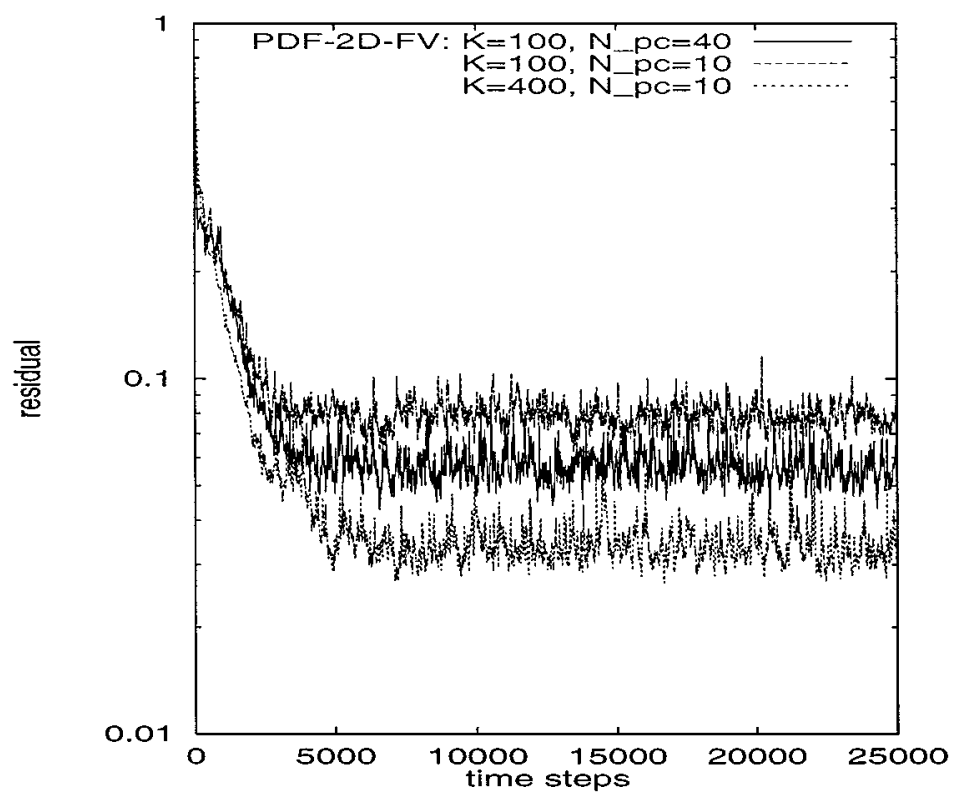

FIG. 6. $P D F-2 D-F V$ with a $40 \times 40$ grid: Residuals in the finite-volume code as a function of time for different time averaging factors $K$ and numbers of particles per cell $N_{p c}$.

\subsection{Bias Error}

The bias error is the deterministic error caused by using a finite number of particles. Simulations using the same $40 \times 40$ grid, but with different $N_{p c}$ have been performed. In Fig. 8 Favre averaged velocity and mixture fraction profiles at $40 R_{\text {jet }}$ downstream of the nozzle are plotted (with $K=100$ and $N_{p c}=10,40,160$ ). One can see that there is a fixed point in each plot where the three lines cross each other. The vertical lines in Fig. 8 mark two locations 1 and $2.5 R_{\text {jet }}$ away from the symmetry axis (left and right of the fix-points)
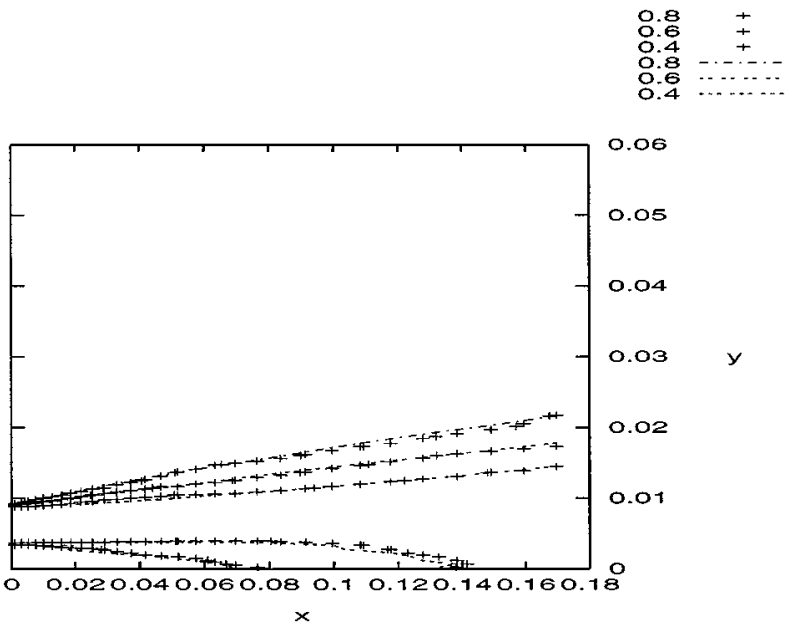

FIG. 7. $P D F-2 D-F V$ with a $40 \times 40$ grid, $K=800$, and $N_{p c}=10$ : Mean density contour plots from the finite-volume data (lines) and extracted from the particle field (symbols). 

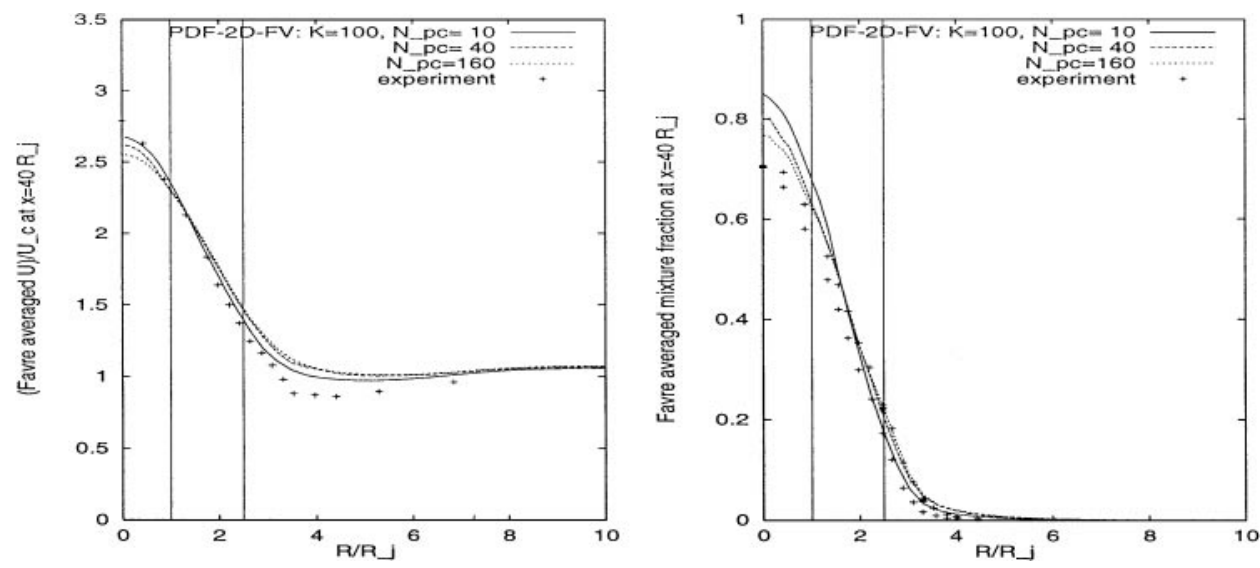

FIG. 8. $P D F-2 D-F V$ with a $40 \times 40$ grid and $K=100$ : Comparison of Favre averaged velocity and mixture fraction profiles at $x=40 R_{\text {jet }}$ for different values of $N_{p c}$.

which we chose to study the asymptotic behavior of the algorithm, as $N_{p c}$ is increased. The same points have been chosen by Xu and Pope [49] to study the bias error of the stand-alone particle-mesh algorithm.

Figure 9 shows the asymptotic behavior (at these two points) of the quantities $\tilde{U}_{1} / U_{\text {coflow }}$,

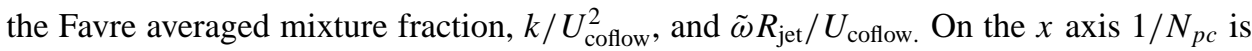
shown, and the points represent the values ( $K=100$ and $\left.N_{p c}=10,20,40,80,160\right)$. The long lines are the least-squares lines (using these data points), and the short lines are the corresponding least-squares lines of the studies with the stand-alone particle-mesh method $P D F 2 D V$. The intersection of these lines with the ordinate is the extrapolation to $N_{p c}^{-1}=0$, i.e, the bias-free result corresponding to an infinite number of particles. The slopes of the lines indicate the magnitude of the bias error. One can see that the short lines are much steeper, which shows that, for a given $N_{p c}$ (and $K=100$ ), the new hybrid scheme leads to a much smaller bias error.

Also shown are the least-squares lines of the results obtained by increasing $K$ instead of increasing $N_{p c}\left(N_{p c}=10\right.$ and $\left.K=100,200,400,800,1600\right)$. These lines are almost identical with the least-squares lines using the values of the results with $K=100$ and $N_{p c}=10, N_{p c}=20, N_{p c}=40, N_{p c}=80$, and $N_{p c}=160$, which shows that multiplying $K$ by a factor $b$ has the same effect on the bias error as multiplying $N_{p c}$ by $b$. The vertical line in the last plot in Fig. 9 shows how many particles are needed to obtain less than $5 \%$ bias error (notice that the relative bias error of $k$ is larger than the bias error of the first moments). This can be achieved with $K=100$ and $N_{p c}=55$ or with $K=550$ and $N_{p c}=10$. For the same error tolerance at the same locations more than 1400 particles are necessary with the stand-alone particle-mesh method. With $K=550$ and $N_{p c}=10$ the hybrid scheme converges in about 7000 time steps, which means that 70,000 particle time steps per cell have to be performed. The stand-alone particle-mesh method was shown to converge in about 1000 time steps, and therefore 1,400,000 particle time steps have to be computed for each cell. From this and the required CPU time (about 1 hour for the hybrid scheme on a $400-\mathrm{MHz}$ Pentium machine) we conclude that the new algorithm is more than 20 times more efficient than the stand-alone particle-mesh method (for this test case). No attempt has been made yet to optimize the choice of $K$ and $N_{p c}$ for a given $C_{\text {bias }}=N_{p c} K$. 

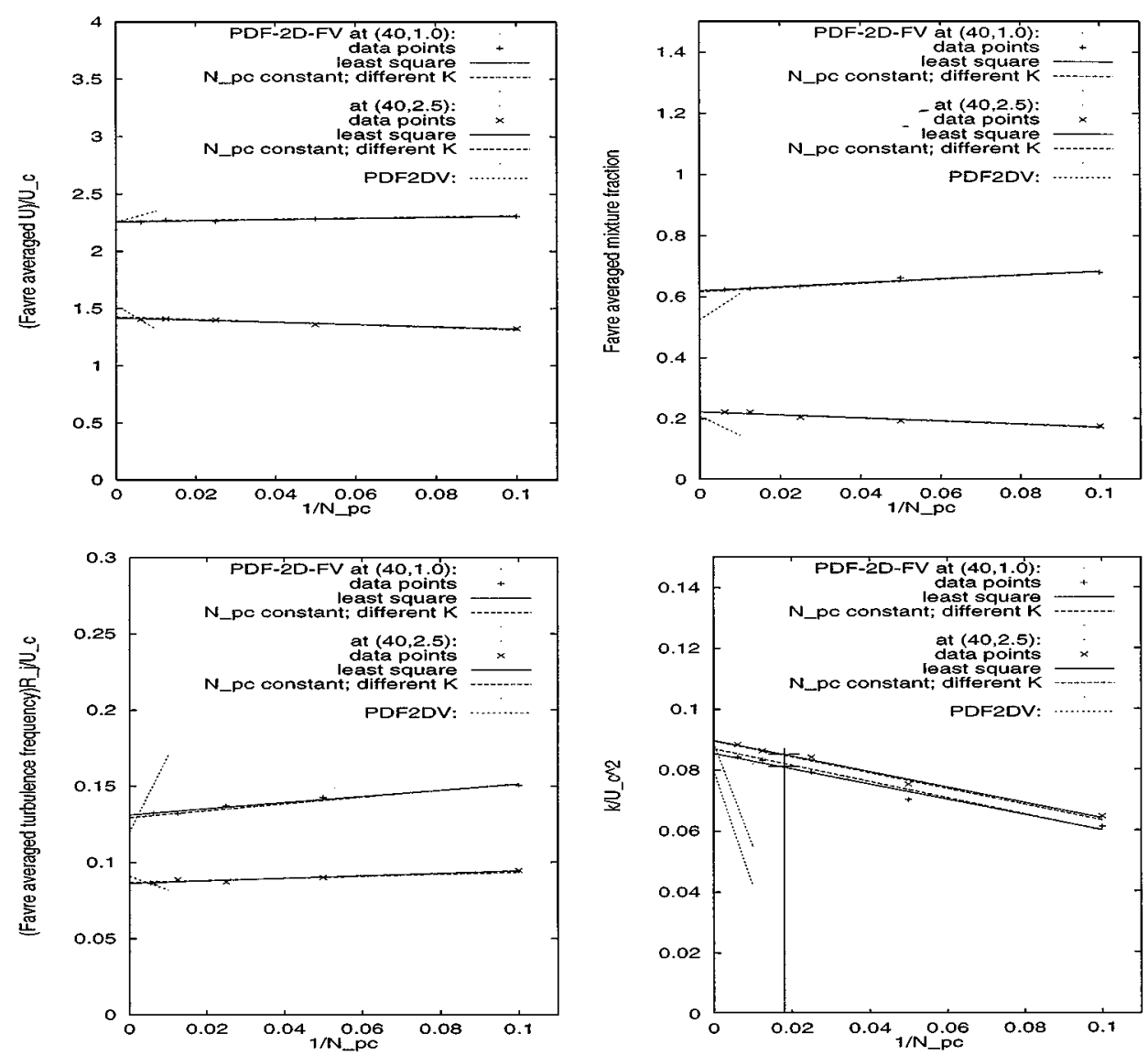

FIG. 9. Bias convergence studies: Mean quantities against $N_{p c}^{-1}$ at different locations for $P D F 2 D V$ and $P D F$ $2 D-F V$.

\subsection{Spatial Discretization Error}

The spatial discretization error is the deterministic error caused by using a finite number of grid cells. Here we show that the hybrid algorithm is second-order accurate in space and that its asymptotic behavior is comparable to that of the stand-alone particle-mesh method. Simulations with $K=100$ and $N_{p c}=10, N_{p c}=20, N_{p c}=40$, and $N_{p c}=80$ have been performed on grids with $20 \times 20,30 \times 30,40 \times 40$, and $50 \times 50$ cells. The same quantities and the same locations as for the convergence studies of the bias error are used here to study the asymptotic behavior of the scheme as the grid is refined. The $x$ axis in Fig. 10 represents $M^{-2}$, and the data points represent the asymptotic values for $N_{p c} \rightarrow \infty$ on the different grids. The long lines are the least-squares lines of these data points on the grids with $30 \times 30,40 \times 40$, and $50 \times 50$ cells. The short lines are the corresponding leastsquares lines of the studies with the stand-alone particle-mesh method. We see that the hybrid algorithm (like the stand-alone particle method) is second-order accurate in space and shows grid convergence rates comparable to those of the particle method. For the mean quantities $\tilde{\mathbf{U}}_{1}$ and $\tilde{\xi}$, there is good agreement between the extrapolated values $\left(N_{p c} \rightarrow \infty, M \rightarrow \infty\right)$ obtained with the two codes. This shows that, although the numerical errors in the two codes have quite different behaviors, they converge to the same result as $N_{p c}$ and $M$ tend to infinity. 

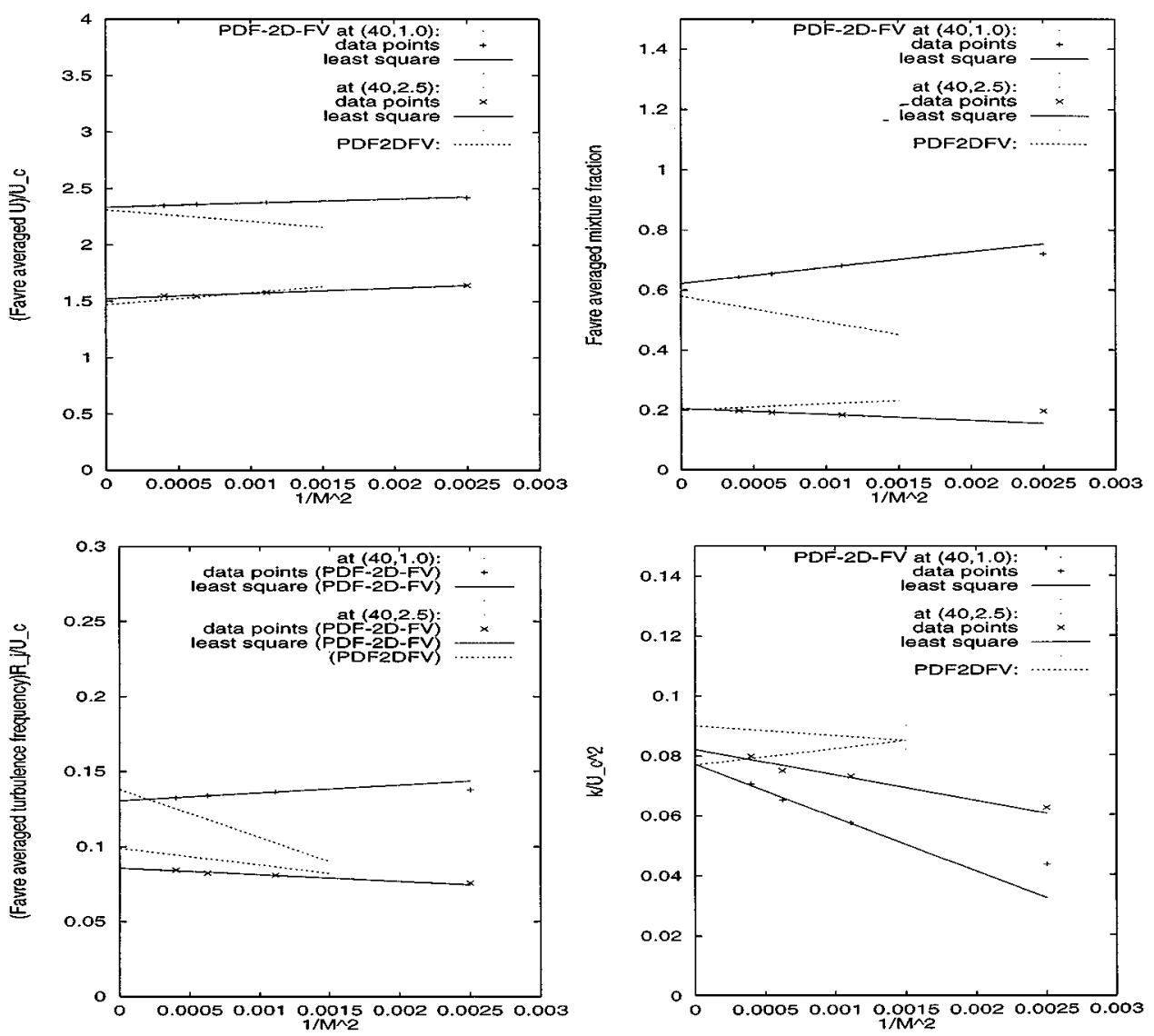

FIG. 10. Grid convergence studies: Mean quantities against $M^{-2}$ at different locations for $P D F 2 D V$ and $P D F-2 D-F V$.

For $\tilde{\omega}$ and $k$ the agreement is less satisfactory. Possible reasons for these discrepancies are:

- On each grid the bias free result is an extrapolated value (and hence subject to extrapolation error).

- The grid converged results are extrapolated values of the bias free results on different grids (and hence subject to extrapolation error).

- The results still contain statistical error.

\section{CONCLUSIONS}

A new hybrid algorithm for solving the joint velocity-frequency-composition PDF transport equation has been developed and implemented in the code $P D F-2 D-F V$. The important aspects of this algorithm are:

- It solves the joint velocity-frequency-composition PDF evolution equation for turbulent reactive flow.

- It is a combination of a finite-volume scheme and a particle method. 
- The modeled equations are consistent.

- It is internally consistent and robust.

- It reaches a statistically stationary state.

- It converges for $N_{p c} \rightarrow \infty$.

- It converges for $M \rightarrow \infty$.

Some of the issues which had to be addressed are listed below.

- Internal consistency: The interpolation of the Favre averaged velocity field $\tilde{\mathbf{U}}$ from the finite-volume data to the particle positions, such that $\nabla \cdot \tilde{\mathbf{U}}$ behaves properly, is crucial to obtaining consistent $\langle\rho\rangle$ and $\tilde{e}_{s}$ fields.

- Stable coupling between the finite-volume scheme and the particle method: This is achieved by using a time-averaging technique.

- Reduction of the statistical and bias error: Instead of the $\langle\rho\rangle$ and $\tilde{\mathbf{U}}$ fields extracted from the particles, the corresponding smoother fields, computed by the finite-volume scheme, are used in the particle method. Furthermore the bias and statistical errors are reduced enormously because of a time-averaging technique.

The computational effort is considered in two parts: that required directly in computing the composition change because of reactions, and that required in the remainder of the PDF particle method. For the latter part, both the work and the storage scale linearly with the number of species. For the reaction part, the work depends on the complexity of the chemistry, its stiffness, and how it is implemented.

Numerical experiments of a nonpremixed piloted-methane-jet flame have been performed to compare the accuracy and efficiency of the new algorithm with the established stand-alone particle-mesh method PDF2DV. (Studies of a bluff-body stabilized flow with different PDF algorithms, including the new hybrid algorithm, can be found in [16].) The conclusions from these studies are the following:

- The converged results $\left(N_{p c} \rightarrow \infty\right.$ and $\left.M \rightarrow \infty\right)$ with $P D F-2 D-F V$ are in good agreement with those of PDF2DV.

- For a given error tolerance $P D F-2 D-F V$ is much more efficient than $P D F 2 D V$ (more than 20 times faster for the nonpremixed piloted-methane-jet flame test case). This is mainly due to the smaller bias error.

These results are very encouraging for joint velocity-frequency-composition PDF methods to be applied for complex 3D flow in the future.

\section{APPENDIX A: VELOCITY FIELD INTERPOLATION}

In Section 5.7 it has been pointed out that the dilatation field $\nabla \cdot \tilde{\mathbf{U}}$ experienced by the particles is of fundamental importance in particle methods and that it makes most sense to use the mean velocities at the volume interfaces (computed by the flux solver) for the interpolation to the particle positions.

Next the 2D interpolation scheme used in our algorithm is derived. First we transform the rectangular cells into unit squares. The axes of the new coordinate system are denoted by $x_{1}^{\prime}$ and $x_{2}^{\prime}$ and their origin is at the bottom left corner of the transformed cell (Fig. 11). Consider a representation of $U_{1}$ that is quadratic in $x_{1}^{\prime}$ and linear in $x_{2}^{\prime}$, and correspondingly 


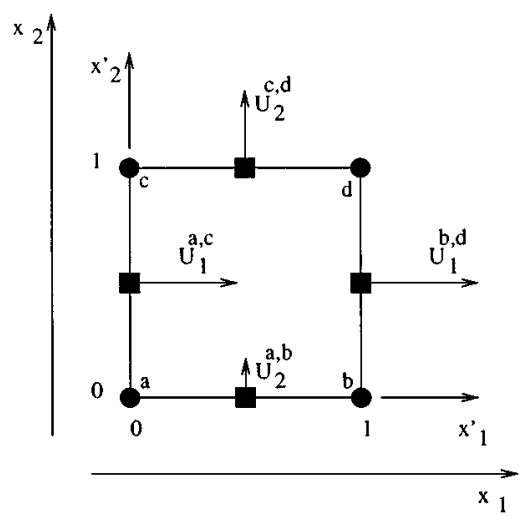

FIG. 11. For velocity field interpolation: Transformed volume.

a representation of $U_{2}$ that is quadratic in $x_{2}^{\prime}$ and linear in $x_{1}^{\prime}$ :

$$
\begin{aligned}
U_{1}\left(x_{1}, x_{2}\right)= & \left(1-x_{2}^{\prime}\right)\left(\left(1-x_{1}^{\prime}\right) U_{1}^{a}+x_{1}^{\prime} U_{1}^{b}-\frac{1}{2} x_{1}^{\prime}\left(1-x_{1}^{\prime}\right) \hat{U}_{1}^{e}\right) \\
& +x_{2}^{\prime}\left(\left(1-x_{1}^{\prime}\right) U_{1}^{c}+x_{1}^{\prime} U_{1}^{d}-\frac{1}{2} x_{1}^{\prime}\left(1-x_{1}^{\prime}\right) \hat{U}_{1}^{f}\right)
\end{aligned}
$$

and

$$
\begin{aligned}
U_{2}\left(x_{1}, x_{2}\right)= & \left(1-x_{1}^{\prime}\right)\left(\left(1-x_{2}^{\prime}\right) U_{2}^{a}+x_{2}^{\prime} U_{2}^{c}-\frac{1}{2} x_{2}^{\prime}\left(1-x_{2}^{\prime}\right) \hat{U}_{2}^{g}\right) \\
& +x_{1}^{\prime}\left(\left(1-x_{2}^{\prime}\right) U_{2}^{b}+x_{2}^{\prime} U_{2}^{d}-\frac{1}{2} x_{2}^{\prime}\left(1-x_{2}^{\prime}\right) \hat{U}_{2}^{h}\right) .
\end{aligned}
$$

The gradients $\partial U_{1} / \partial x_{1}$ and $\partial U_{2} / \partial x_{2}$ are then given by

$$
\begin{aligned}
\Delta x_{1} \frac{\partial U_{1}}{\partial x_{1}}= & \left(1-x_{2}^{\prime}\right)\left(-U_{1}^{a}+U_{1}^{b}+\left(x_{1}^{\prime}-\frac{1}{2}\right) \hat{U}_{1}^{e}\right) \\
& +x_{2}^{\prime}\left(-U_{1}^{c}+U_{1}^{d}+\left(x_{1}^{\prime}-\frac{1}{2}\right) \hat{U}_{1}^{f}\right)
\end{aligned}
$$

and

$$
\begin{aligned}
\Delta x_{2} \frac{\partial U_{2}}{\partial x_{2}}= & \left(1-x_{1}^{\prime}\right)\left(-U_{2}^{a}+U_{2}^{c}+\left(x_{2}^{\prime}-\frac{1}{2}\right) \hat{U}_{2}^{g}\right) \\
& +x_{1}^{\prime}\left(-U_{2}^{b}+U_{2}^{d}+\left(x_{2}^{\prime}-\frac{1}{2}\right) \hat{U}_{2}^{h}\right) .
\end{aligned}
$$

These representations have the following properties:

1. At node $a, U_{1}\left(x_{1}, x_{2}\right)=U_{1}^{a}$ and $U_{2}\left(x_{1}, x_{2}\right)=U_{2}^{a}$, and similarly for the other three corners.

2. The velocities normal to the cell faces (i.e., $U_{1}$ at the east and west faces, $U_{2}$ at the north and south faces) are independent of $\hat{U}_{1}^{e}, \hat{U}_{1}^{f}, \hat{U}_{2}^{g}$ and $\hat{U}_{2}^{h}$. 
To determine the coefficients $\hat{U}_{1}^{e}, \hat{U}_{1}^{f}, \hat{U}_{2}^{g}$, and $\hat{U}_{2}^{h}$ we require that $\nabla \cdot \mathbf{U}$ vary linearly within the cell. At the four corners we have

$$
\begin{aligned}
& (\nabla \cdot \mathbf{U})^{a}=\left(-U_{1}^{a}+U_{1}^{b}-\frac{1}{2} \hat{U}_{1}^{e}\right) / \Delta x_{1}+\left(-U_{2}^{a}+U_{2}^{c}-\frac{1}{2} \hat{U}_{2}^{g}\right) / \Delta x_{2} \\
& (\nabla \cdot \mathbf{U})^{b}=\left(-U_{1}^{a}+U_{1}^{b}+\frac{1}{2} \hat{U}_{1}^{e}\right) / \Delta x_{1}+\left(-U_{2}^{b}+U_{2}^{d}-\frac{1}{2} \hat{U}_{2}^{h}\right) / \Delta x_{2} \\
& (\nabla \cdot \mathbf{U})^{c}=\left(-U_{1}^{c}+U_{1}^{d}-\frac{1}{2} \hat{U}_{1}^{f}\right) / \Delta x_{1}+\left(-U_{2}^{a}+U_{2}^{c}-\frac{1}{2} \hat{U}_{2}^{g}\right) / \Delta x_{2} \\
& (\nabla \cdot \mathbf{U})^{d}=\left(-U_{1}^{c}+U_{1}^{d}+\frac{1}{2} \hat{U}_{1}^{f}\right) / \Delta x_{1}+\left(-U_{2}^{b}+U_{2}^{d}-\frac{1}{2} \hat{U}_{2}^{h}\right) / \Delta x_{2} .
\end{aligned}
$$

Note that the average of the above four expressions is

$$
\frac{\frac{1}{2}\left(U_{1}^{b}+U_{1}^{d}-U_{1}^{a}-U_{1}^{c}\right)}{\Delta x}+\frac{\frac{1}{2}\left(U_{2}^{c}+U_{2}^{d}-U_{2}^{a}-U_{2}^{b}\right)}{\Delta y}
$$

independent of $\hat{U}_{1}^{e}, \hat{U}_{1}^{f}, \hat{U}_{2}^{g}$ and $\hat{U}_{2}^{h}$. Our algorithm to determine the velocity components at the volume corners and the coefficients $\hat{U}_{1}^{e}, \hat{U}_{1}^{f}, \hat{U}_{2}^{g}$ and $\hat{U}_{2}^{h}$ is:

1. To make (59) consistent with the FV part of the code, the velocity components at the corners of the volumes are chosen such that

$$
\begin{aligned}
& \frac{1}{2}\left(U_{2}^{a}+U_{2}^{b}\right)=U_{2}^{a, b} \\
& \frac{1}{2}\left(U_{2}^{c}+U_{2}^{d}\right)=U_{2}^{c, d} \\
& \frac{1}{2}\left(U_{1}^{a}+U_{1}^{c}\right)=U_{1}^{a, c} \\
& \frac{1}{2}\left(U_{1}^{b}+U_{1}^{d}\right)=U_{1}^{b, d},
\end{aligned}
$$

where $U_{1}^{a, c}$ is the $x_{1}$ velocity component of the mean flux (used in the finite-volume scheme) at the corresponding volume interface, and similarly for the other component and the other interfaces.

2. There are different possible choices for the velocity components at the volume corners to fulfill (60). To avoid oscillations we allow different values at a specific volume corner; i.e., we use a linear interpolation technique combined with the minmod limiter (Fig. 12) such that (60) is fulfilled:

For example, for volume $(i, j)$,

$$
U_{2_{i}}^{b}=U_{2_{i, j}}^{a, b}+\frac{\Delta x_{1_{i, j}}}{2} \operatorname{minmod}\left(\frac{U_{2_{i+1, j}}^{a, b}-U_{2_{i, j}}^{a, b}}{x_{1_{i+1, j}}-x_{1_{i, j}}}, \frac{U_{2_{i, j}}^{a, b}-U_{2_{i-1, j}}^{a, b}}{x_{1_{i, j}}-x_{1_{i-1, j}}}\right),
$$

and for volume $(i+1, j)$,

$$
U_{2_{i+1, j}}^{a}=U_{2_{i+1, j}}^{a, b}-\frac{\Delta x_{1_{i+1, j}}}{2} \operatorname{minmod}\left(\frac{U_{2_{i+2, j}}^{a, b}-U_{2_{i+1, j}}^{a, b}}{x_{1_{i+2, j}}-x_{1_{i+1, j}}}, \frac{U_{2_{i+1, j}}^{a, b}-U_{2_{i, j}}^{a, b}}{x_{1_{i+1, j}}-x_{1_{i, j}}}\right) .
$$




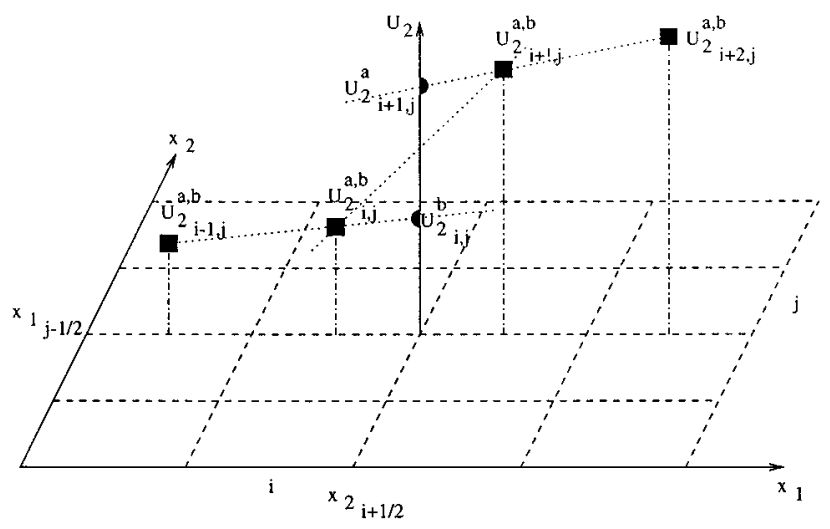

FIG. 12. For velocity field interpolation: MUSCL with minmod limiter; squares indicate velocities obtained from fluxes computed by the finite-volume scheme and circles indicate extrapolated values; note that $U_{2_{i+1, j}}^{a} \neq U_{2_{i, j}}^{b}$ although they are defined at the same location.

The minmod limiter is defined as

$$
\operatorname{minmod}(A, B)=\left\{\begin{array}{llll}
A & \text { if } A \cdot B>0 & \text { and } & |A| \leq|B| \\
B & \text { if } A \cdot B>0 & \text { and } & |A|>|B| \\
0 & \text { if } A \cdot B \leq 0 &
\end{array}\right.
$$

Note that in general $U_{2_{i+1, j}}^{a} \neq U_{2_{i, j}}^{b}$ although corner a of volume $(i+1, j)$ has the same location as corner $b$ of volume $(i, j)$, but the difference $U_{2_{i+1, j}}^{a}-U_{2_{i, j}}^{b}$ is proportional to $1 / M^{2}$ when the velocity field is smooth [44].

3. At the corner $a, d^{\prime a}$ is defined as the average of $\nabla \cdot \tilde{\mathbf{U}}$ over the four incident cells and is used as an initial estimate for $(\nabla \cdot \tilde{\mathbf{U}})^{a}$. Similarly, $d^{\prime b}, d^{\prime c}$, and $d^{\prime d}$ are defined at the other three corners.

4. For a given cell the additive adjustment

$$
\delta=\frac{U_{1}^{b}+U_{1}^{d}-U_{1}^{a}-U_{1}^{c}}{2 \Delta x_{1}}+\frac{U_{2}^{c}+U_{2}^{d}-U_{2}^{a}-U_{2}^{b}}{2 \Delta x_{2}}-\frac{d^{\prime a}+d^{\prime b}+d^{\prime c}+d^{\prime d}}{4}
$$

is made to $(\nabla \cdot \tilde{\mathbf{U}})^{a, b, c, d}$ in order that the average of the modified values

$$
(\nabla \cdot \tilde{\mathbf{U}})^{a, b, c, d}=d^{a, b, c, d}=d^{\prime a, b, c, d}+\delta
$$

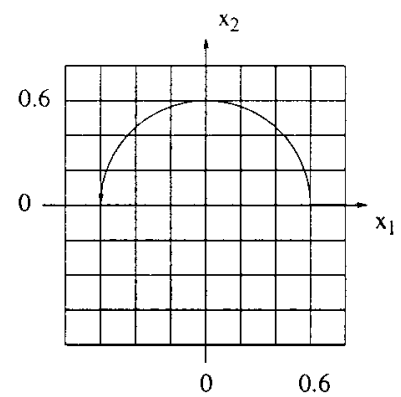

FIG. 13. Particle tracking test case: To test the accuracy of the velocity interpolation scheme. 
satisfies (59). Notice that $\delta$ and $d^{a, b, c, d}$ are only used for the derivation of coefficients $\hat{U}$, but not for the implementation.

5. Given $U_{1,2}^{a, b, c, d}$ from Eqs. (61) and (62) and $(\nabla \cdot \tilde{\mathbf{U}})^{a, b, c, d}$ from Eq. (65), the linearity of $\nabla \cdot \mathbf{U}(58)$ imposes three independent constraints on the four remaining coefficients $\hat{U}_{1}^{e}, \hat{U}_{1}^{f}, \hat{U}_{2}^{g}$, and $\hat{U}_{2}^{h}$. We specify these coefficients as the least-squares solution

$$
\begin{aligned}
\hat{U}_{1}^{e}= & \frac{\left(-d^{\prime a}+d^{\prime b}-d^{\prime c}+d^{\prime d}\right) \frac{\Delta x_{1}^{3}}{2}+\left(d^{\prime b}-d^{\prime a}\right) \Delta x_{1} \Delta x_{2}^{2}}{\Delta x_{1}^{2}+\Delta x_{2}^{2}} \\
& -\frac{\Delta x_{1}}{\Delta x_{2}}\left(U_{2}^{a}-U_{2}^{b}-U_{2}^{c}+U_{2}^{d}\right) \\
\hat{U}_{1}^{f}= & \frac{\left(-d^{\prime a}+d^{\prime b}-d^{\prime c}+d^{\prime d}\right) \frac{\Delta x_{1}^{3}}{2}+\left(d^{\prime d}-d^{\prime c}\right) \Delta x_{1} \Delta x_{2}^{2}}{\Delta x_{1}^{2}+\Delta x_{2}^{2}} \\
& -\frac{\Delta x_{1}}{\Delta x_{2}}\left(U_{2}^{a}-U_{2}^{b}-U_{2}^{c}+U_{2}^{d}\right) \\
\hat{U}_{2}^{g}= & \frac{\left(-d^{\prime a}-d^{\prime b}+d^{\prime c}+d^{\prime d}\right) \frac{\Delta x_{2}^{3}}{2}+\left(d^{\prime c}-d^{\prime a}\right) \Delta x_{2} \Delta x_{1}^{2}}{\Delta x_{1}^{2}+\Delta x_{2}^{2}} \\
& -\frac{\Delta x_{2}}{\Delta x_{1}}\left(U_{1}^{a}-U_{1}^{b}+U_{1}^{c}+U_{1}^{d}\right) \\
\hat{U}_{2}^{h}= & \frac{\left(-d^{\prime a}-d^{\prime b}+d^{\prime c}+d^{\prime d}\right) \frac{\Delta x_{2}^{3}}{2}+\left(d^{\prime b}-d^{\prime b}\right) \Delta x_{2} \Delta x_{1}^{2}}{\Delta x_{1}^{2}+\Delta x_{2}^{2}} \\
& -\frac{\Delta x_{2}}{\Delta x_{1}}\left(U_{1}^{a}-U_{1}^{b}-U_{1}^{c}+U_{1}^{d}\right),
\end{aligned}
$$

which satisfies (58) and minimizes

$$
\left(\hat{U}_{1}^{e}\right)^{2}+\left(\hat{U}_{1}^{f}\right)^{2}+\left(\hat{U}_{2}^{g}\right)^{2}+\left(\hat{U}_{2}^{h}\right)^{2}
$$

In our case this scheme is applied in order to interpolate the mean velocity field $\tilde{\mathbf{U}}$. In summary, within each cell, the mean velocity is given by (54) and (55), with the coefficients determined from the finite-volume cell-face velocities from (61), (62), and (66). Within each cell, $\nabla \cdot \tilde{\mathbf{U}}$ varies bilinearly with $x_{1}$ and $x_{2}$ (for constant density flow, $\nabla \cdot \tilde{\mathbf{U}}=0$ everywhere).

At the cell faces, the longitudinal velocity is discontinuous (because of the use of the minmod limiter and because the coefficients $\hat{U}_{1}^{e}, \hat{U}_{1}^{f}, \hat{U}_{2}^{g}$, and $\hat{U}_{2}^{h}$ are different in each cell). For a smooth field, these velocity discontinuities tend to zero as $\Delta x^{2}$.

Across cell faces $\nabla \cdot \tilde{\mathbf{U}}$ is discontinuous because of the adjustment $\delta$ (64), which also decreases as $\Delta x^{2}$.

\section{Test Case: Particle Tracking}

This case was to test the spatial accuracy of the velocity interpolation scheme. A number of particles are tracked in a given flow field (nonconstant density). All variables are nondimensionalized by a reference value of one in the corresponding SI units. The domain is the 


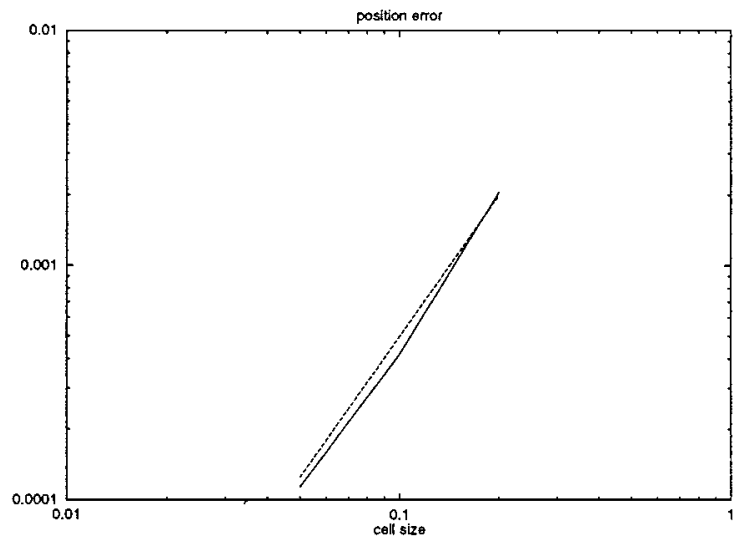

FIG. 14. Accuracy of the velocity interpolation scheme: Particle position error as a function of grid cell size.

2D plane with $x_{2}>-2$, the velocity field is given by

$$
\langle\rho\rangle\left(\begin{array}{c}
\tilde{U}_{1} \\
\tilde{U}_{2}
\end{array}\right)=\frac{1}{\left(x_{1}^{2}+x_{2}^{2}\right)^{1 / 2}}\left(\begin{array}{c}
-x_{2} \\
x_{1}
\end{array}\right)
$$

and the density field is

$$
\langle\rho\rangle=2+x_{2}
$$

The streamlines are circles, and the continuity equation is satisfied. For the interpolation we used the mean velocities and the mean densities at the volume interfaces of a uniform rectangular grid. It takes $2 r \alpha+r^{2}(1-\cos (\alpha))$ seconds for a particle with the initial position $(r, 0)$ to get to the position $(r \cos (\alpha), r \sin (\alpha))$. We tracked 50 particles, initially uniformly distributed on the line from $(0.6,0)$ to $(0.65,0)$, and chose the time $t$ such that the most inner particle, i.e., the particle with the initial position $(0.6,0)$, has the final position $(-0.6$, 0 ). In Fig. 14 the mean position errors for three different grids are shown (with cell size 0.2, 0.1 , and 0.05 , respectively). The dashed line is for reference of exactly second oder.

\section{APPENDIX B: TIME AVERAGING}

The purpose of this appendix is to analyze a time-averaging technique for coupling the finite-volume scheme with the particle method such that the overall scheme is stable. Furthermore this time-averaging technique reduces the statistical error, and since all the terms in the SDEs and in the finite-volume scheme which are extracted from the particle field are time averaged, the bias error also is reduced.

For the finite-volume scheme we expect the mean residual $\sigma^{n}$ on the $n$th step to decay as

$$
\sigma^{n+1}=v \sigma^{n} \quad(\text { for stable schemes } 0<v<1) .
$$

Now we consider the model system, which at time step $n$ is characterized by $\gamma^{n}$ (which represents the finite-volume data) and $\tilde{\beta}^{n}$ (which represents the the time-averaged extracted 
particle method data)

$$
\begin{aligned}
& \gamma^{n+1}=v \gamma^{n}+\tilde{\beta}^{n} \\
& \tilde{\beta}^{n+1}=\mu \tilde{\beta}^{n}+(1-\mu) \tilde{\beta}_{i}^{n+1} \quad \text { with }(0 \leq \mu<1),
\end{aligned}
$$

where $\tilde{\beta}_{i}^{n}$ is the instantaneous extracted particle method data. Equation (71) represents the finite-volume scheme, and Eq. (72) is the time-averaging scheme (52) with the factor $\mu=\frac{K-1}{K}$, where $K$ is the time-averaging factor. Defining $\alpha^{n}=\gamma^{n+1}-\gamma^{n}$ and using (71) we can write

$$
\alpha^{n+1}=v \alpha^{n}+\left(\tilde{\beta}^{n+1}-\tilde{\beta}^{n}\right)
$$

and defining $\tilde{\beta}_{i}^{n+1}=a+b \alpha^{n}+c \xi^{n+1}$ and $\delta^{n}=a-\tilde{\beta}^{n}$ (the expectation of $\tilde{\beta}_{i}^{n}$ converges toward a constant $a$, has a statistical error which is proportional to a constant $c$, and is linearly dependent on $\alpha^{n}$ ), one can write

$$
\delta^{n+1}=(-b(1-\mu)) \alpha^{n}+\mu \delta^{n}-c(1-\mu) \xi^{n+1} .
$$

Finally we get the model system

$$
\left(\begin{array}{l}
\delta \\
\alpha
\end{array}\right)^{n+1}=\underbrace{\left(\begin{array}{cc}
\mu & -b(1-\mu) \\
1-\mu & \nu+b(1-\mu)
\end{array}\right)}_{\underline{\mathbf{A}}}\left(\begin{array}{c}
\delta \\
\alpha
\end{array}\right)^{n}+c(1-\mu)\left(\begin{array}{c}
-1 \\
1
\end{array}\right) \xi^{n+1} .
$$

Neglecting the nondeterministic term (i.e., setting $c=0$ ) we have a linear system with $|\underline{\mathbf{A}}|=\mu(v-b)+b$. The eigenvalues $\lambda_{1,2}$ of $\underline{\mathbf{A}}$ are

$$
\begin{aligned}
\lambda_{1,2}= & \frac{1}{2}(\mu+b-b \mu+v) \pm \frac{1}{2}\left(\mu^{2}+6 b \mu-2 b \mu^{2}-2 \mu v+b^{2}\right. \\
& \left.-2 b^{2} \mu+2 v b+b^{2} \mu^{2}-2 b \mu v+v^{2}-4 b\right)^{\frac{1}{2}}
\end{aligned}
$$

and can be complex or real. In Figs. 15, 16, and 17, $\left|\lambda_{2}\right|$ (the greater of the absolute values of the two eigenvalues) is plotted as a function of $\mu$ for $v=0.99$ and different values of

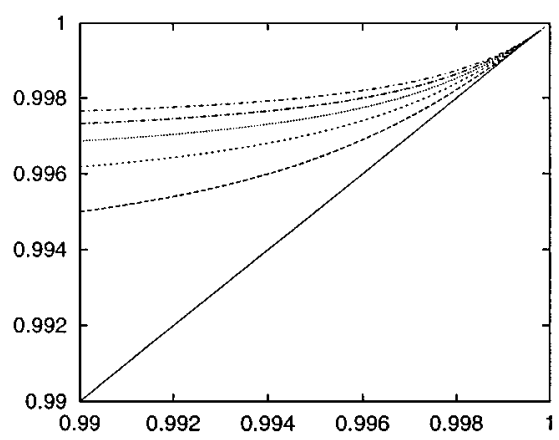

FIG. 15. Model system (75): $\left|\lambda_{2}\right|$ as a function of $\mu$ for $v=0.99$ and $b<0$. 


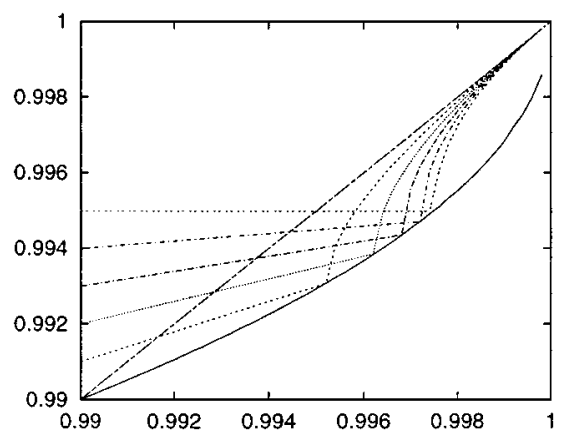

FIG. 16. Model system (75): $\left|\lambda_{2}\right|$ as a function of $\mu$ for $v=0.99$ and $0<b<v$.

$b \leq 0, v \geq b \geq 0$ and $b \geq v$, respectively. Furthermore, for reference, $\left|\lambda_{\text {ref }}\right|=\mu$ is shown. It can be seen that the critical family is the one shown in Fig. $17(b \geq v)$. In that family the best choice of $\mu$ is the value for which the eigenvalues $\lambda_{1,2}$ change their nature from complex to real. One can think about different strategies for finding the optimal time-averaging factor $K=\frac{1}{1-\mu}$. At the moment, we make no attempt in this direction. We choose an appropriate value for $\mu$ which is smaller than one, but large enough to have a stable scheme. To illustrate the convergence behavior of the model system (75), Fig. 18 shows $\log \left|\alpha^{n}\right|$ for $b=1.5, c=0.0001, v=0.99$, and three different values of $\mu$. While the system is unstable for $\mu=0.97$, the best convergence rate is found for $\mu=0.9979795896$. The third convergence plot is obtained with $\mu=v=0.99$. For reference the convergence history line of the system with $\beta^{n} \equiv 0$ is shown. The horizontal lines represent the value $c(1-\mu)$ for $\mu=0.9979795896$ and $\mu=0.99$. This is the statistical error where the convergence stalls.

Unfortunately the coupled finite-volume/particle method algorithm is much more complex than the studied model system. It is in general not straightforward to determine the parameters $v$ and $b$, and therefore it is difficult, if not impossible, to find the optimal choice for the factor $\mu$. However, we can learn the following from the previous studies:

- The question of how well the coupled system converges (and if stable or not) depends not on the amplitude of noise (parameter $c$ in the model system (75)), but on the convergence rate $v$ of the stand-alone finite-volume scheme, on the sensitivity of $\tilde{\beta}_{i}$ on $\gamma$ (parameter $b$ in the model system (71), (72)), and on the choice of the time-averaging factor $K=\frac{1}{1-\mu}$.

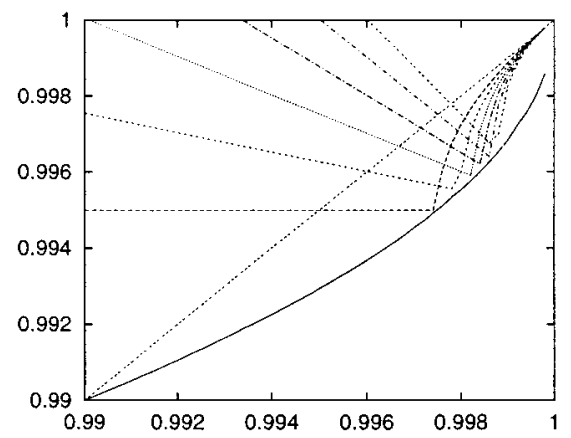

FIG. 17. Model system (75): $\left|\lambda_{2}\right|$ as a function of $\mu$ for $v=0.99$ and $b \geq v$. 


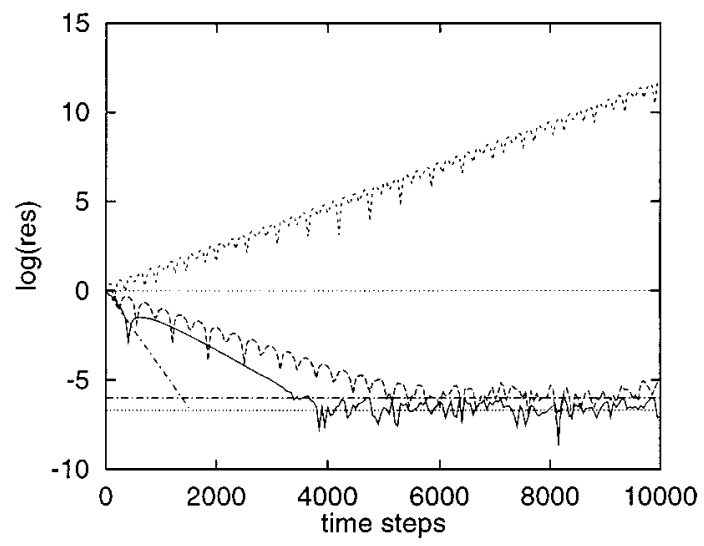

FIG. 18. Convergence histories of the model system (75): $v^{n}$ (steepest descending line; stand-alone finitevolume) and $\log \left|\alpha^{n}\right|$ for $b=1.5, c=0.0001, v=0.97$ (climbing curve; unstable), $v=0.99$ (dashed line; stable, but not optimal), and $\mu=0.9979795896$ (solid line; optimal). The lowest two horizontal lines represent the value $c(1-\mu)$ for $\mu=0.9979795896$ and $\mu=0.99$ (statistical error where the convergence stalls).

- One can always find a value for $\mu$ such that the overall convergence rate $v_{\text {overall }}\left(\left|\lambda_{2}\right|\right.$ in the model system (75)) is less than one.

- The convergence stalls, if the residual reaches $O(c(1-\mu))$.

\section{ACKNOWLEDGMENTS}

This work has been supported in part by the Swiss National Foundation for young scientists, by Department of Energy Grant DE-FG02-90ER-14128, and by Air Force Office of Scientific Research Grant F49620-97-1-0126.

\section{REFERENCES}

1. M. S. Anand, A. T. Hsu, and S. B. Pope, Calculations of swirl combustors using joint velocity-scalar probability density function method, AIAA J. 35, 1143-1150 (1997).

2. G.-C. Chang, A Monte Carlo PDF/ Finite-volume Study of Turbulent Flames, Ph.D. thesis (Cornell University, 1996).

3. J. Y. Chen, W. Kollmann, and R. W. Dibble, Pdf modeling of turbulent nonpremixed methane jet flames. Combust. Sci. Technol. 64, 315-346 (1989).

4. P. J. Colucci, F. A. Jaberi, P. Givi, and S. B. Pope, Filtered density function for large eddy simulation of turbulent reacting flows, Phys. Fluids 10, 499-515 (1998).

5. S. M. Correa and S. B. Pope, Comparison of a Monte Carlo PDF finite-volume mean flow model with bluffbody Raman data, in Twenty-Fourth Symposium (Int'l) on Combustion (Combustion Institute, Pittsburgh, 1992), pp. 279-285.

6. B. J. Delarue and S. B. Pope, Application of PDF methods to compressible turbulent flows, Phys. Fluids $\mathbf{9}$, 2704-2715 (1997).

7. B. J. Delarue and S. B. Pope, Calculations of subsonic and supersonic turbulent reacting mixing layers using probability density function methods, Phys. Fluids 10, 487-498 (1998).

8. C. Dopazo, Recent developments in pdf methods, in Turbulent Reacting Flows, edited by P. A. Libby and F. A. Williams (Academic Press, London, 1994), chapter 7, pp. 375-474.

9. T. D. Dreeben and S. B. Pope, PDF/Monte Carlo simulation of near-wall turbulent flows, J. Fluid Mech. 357, 141-166 (1998). 
10. D. C. Haworth and S. B. Pope, A generalized Langevin model for turbulent flows, Phys. Fluids 29, 387-405 (1986).

11. R. W. Hockney and J. W. Eastwood, Computer Simulations Using Particles (Adam Hilger, (1988).

12. Jayesh and S. B. Pope, Stochastic Model for Turbulent Frequency, Unpublished Technical Report FDA 95-05 (Cornell University, 1995).

13. P. Jenny, On the Numerical Solution of the Compressible Navier-Stokes Equations for Reacting and NonReacting Gas Mixtures, Ph.D. thesis (Swiss Federal Institute of Technology, 1997).

14. P. Jenny and B. Müller, Convergence acceleration for computing steady state compressible flow at low Mach numbers, Combust. Flame 28, 951-972 (1999).

15. P. Jenny, B. Müller, and H. Thomann, Correction of conservative Euler solvers for gas mixtures, J. Comput. Phys. 132, 91-107 (1996).

16. P. Jenny, M. Muradoglu, K. Liu, S. B. Pope, and D. A. Caughey, Pdf simulations of a bluff-body stabilized flow, submitted for publication.

17. W. P. Jones, Turbulence modelling and numerical solution methods for variable density and combusting flows, in Turbulent Reacting Flows, Edited by P. A. Libby and F. A. Williams (Academic Press, London, 1994), p. 309-.

18. M. H. Kalos and P. A. Whitlock, Monte Carlo Methods (Wiley, New York, 1986).

19. B. E. Launder, Phenomenological modelling: Present...and future? in Whither Turbulence? Turbulence at the Crossroads, Edited by J. L. Lumley, Lecture Notes in Physics (Springer-Verlag, Berlin, 1990), pp. 439-485.

20. B. E. Launder and D. B. Spalding, Mathematical Models of Turbulence (Academic Press, London, 1972).

21. A. R. Masri, Technical Report (The University of Sydney, available at http://www.mech.eng.usyd.eud.au/ research/energy/.

22. A. R. Masri, R. W. Dibble, and R. S. Barlow, The structure of turbulent nonpremixed flames revealed by Raman-Rayleigh-LIF measurements, Prog. Energy Combust. Sci. 22, 307-362 (1996).

23. A. R. Masri, R. W. Dibble, and R. W. Bilger, Turbulent non-premixed flames of methane near extinction: Mean structure from Raman measurements, Combust. Flame (1986).

24. A. R. Masri and S. B. Pope, PDF calculations of piloted turbulent non-premixed flames of methane, Combust. Flame 81, 13-29 (1990).

25. J.-P. Minier and J. Pozorski, Analysis of a PDF model in a mixing layer case, in Tenth Symposium on Turbulent Shear Flows (1995), pp. 26.25-26.30.

26. M. Muradoglu, S. B. Pope, P. Jenny, and D. A. Caughey, A consistent hybrid finite-volume/particle method for the pdf equations of turbulent reactive flows, J. Comput, Phys. 154, 342-371 (1999).

27. P. A. Nooren, H. A. Wouters, T. W. J. Peeters, D. Rockaerts, U. Mass, and D. Schmidt, Monte Carlo pdf simulation of a turbulent natural-gas diffusion flame, in Twenty-Sixth Symposium (Int'l) on Combustion (Combustion Institute, Pittsburgh, 1996).

28. P. Jenny, B. Müller, and H. Thomann, Correction of multidimensional conservative Euler solvers for gas mixtures, in Proceedings of 6th International Sysmposium on Computational Fluid Dynamics, Volume I, Lake Tahoe, 1995, pp. 503-508.

29. S. B. Pope, A Monte Carlo method for the PDF equations of turbulent reactive flow, Combust. Sci. Technol. 25, 159-174 (1981).

30. S. B. Pope, PDF methods for turbulent reactive flows, Prog. Energy Combust. Sci. 11, 119-192 (1985).

31. S. B. Pope, Computations of turbulent combustion: Progress and challenges, in Twenty-Third Symposium (Int'l) on Combustion (Combustion Institute, Pittsburgh, 1990), pp. 591-612.

32. S. B. Pope, Lagrangian PDF methods for turbulent flows, Annu. Rev. Fluid. Mech. 26, $23-63$ (1994).

33. S. B. Pope, On the relationship between stochastic Lagrangian models of turbulence and second-moment closures, Phys. Fluids 6, 973-985 (1994).

34. S. B. Pope, PDF2DV: A Fortran code to solve the modelled joint PDF equations for two-dimensional recirculating flows, Unpublished (Cornell University, 1994).

35. S. B. Pope, Particle method for turbulent flows: Integration of stochastic model equations, J. Comp. Phys. 117, 332-349 (1995). 
36. S. B. Pope, Computationally efficient implementation of combustion chemistry using in situ adaptive tabulation, Combust. Theory Modelling 1, 41-63 (1997).

37. S. B. Pope, The vanishing effect of molecular diffusivity on turbulent dispersion: Implications for turbulent mixing and the scalar flux, J. Fluid Mech. 359, 299-312 (1998).

38. S. B. Pope, Turbulent Flows (Cambridge Univ. Press, Cambridge, UK, 2000).

39. S. B. Pope and Y. L. Chen, The velocity-dissipation probability density function model for turbulent flows, Phys. Fluids A 2, 1437-1449 (1990).

40. P. L. Roe, Upwind Differencing Schemes for Hyperbolic Conservation Laws with Source Terms, Edited by C. Carasso, P. A. Raviart, D. Serre, Lecture Notes in Mathematics 1270 (Springer-Verlag, Berlin, 1987).

41. V. Saxena and S. B. Pope, PDF calculations of major and minor species in a turbulent piloted jet flame, in Twenty-Seventh Symposium (Int'l.) on Combustion (Combustion Institute, Pittsburgh, 1998), pp. 1081-1086.

42. J. Sesterhenn, B. Müller, and H. Thomann. A simple characteristic flux evaluation for subsonic flow, in $2 n d$ ECCOMAS CDF Conference (Wiley, Chichester, 1994), p. 57.

43. S. Subramaniam, PDF Models for Mixing in Turbulent Reactive Flows, Ph.D. thesis (Cornell University, 1997).

44. B. van Leer, Towards the ultimate conservative difference scheme. v. a second order sequel to Godunov's method, J. Comput. Phys. 32, 101 (1979).

45. P. R. Van Slooten, Jayesh, and S. B. Pope, Advances in PDF modeling for inhomogeneous turbulent flows. Phys. Fluids 10, 246-265 (1998).

46. P. R. Van Slooten and S. B. Pope, PDF modeling of inhomogeneous turbulence with exact representation of rapid distortions, Phys. Fluids 9, 1085-1105 (1997).

47. P. R. Van Slooten and S. B. Pope, Application of PDF modeling to swirling and non-swirling turbulent jets, Flow, Turbulence and Combustion 62, 295-333 (1999).

48. D. C. Wilcox, Turbulence Modeling for CFD (DCW Industries, La Cañada, CA, 1993).

49. J. Xu and S. B. Pope, Assessment of numerical accuracy of pdf/Monte Carlo methods for turbulent reactive flows, J. Comp. Phys. 152, 192-230 (1999).

50. J. Xu and S. B. Pope. Pdf calculations of turbulent nonpremixed flames with local extinction, Combust. Flame 123, 281-307 (2000). 